\title{
MISR Dark Water aerosol retrievals: operational algorithm sensitivity to particle non-sphericity
}

\author{
O. V. Kalashnikova, M. J. Garay, J. V. Martonchik, and D. J. Diner \\ Jet Propulsion Laboratory, California Institute of Technology, Pasadena, CA, USA \\ Correspondence to: O. V. Kalashnikova (olga.kalashnikova@jpl.nasa.gov)
}

Received: 19 January 2013 - Published in Atmos. Meas. Tech. Discuss.: 12 February 2013

Revised: 15 June 2013 - Accepted: 18 July 2013 - Published: 27 August 2013

\begin{abstract}
The aim of this study is to theoretically investigate the sensitivity of the Multi-angle Imaging SpectroRadiometer (MISR) operational (version 22) Dark Water retrieval algorithm to aerosol non-sphericity over the global oceans under actual observing conditions, accounting for current algorithm assumptions. Non-spherical (dust) aerosol models, which were introduced in version 16 of the MISR aerosol product, improved the quality and coverage of retrievals in dusty regions. Due to the sensitivity of the retrieval to the presence of non-spherical aerosols, the MISR aerosol product has been successfully used to track the location and evolution of mineral dust plumes from the Sahara across the Atlantic, for example. However, the MISR global non-spherical aerosol optical depth (AOD) fraction product has been found to have several climatological artifacts superimposed on valid detections of mineral dust, including high non-spherical fraction in the Southern Ocean and seasonally variable bands of high non-sphericity. In this paper we introduce a formal approach to examine the ability of the operational MISR Dark Water algorithm to distinguish among various spherical and non-spherical particles as a function of the variable MISR viewing geometry. We demonstrate the following under the criteria currently implemented: (1) Dark Water retrieval sensitivity to particle nonsphericity decreases for AOD below about 0.1 primarily due to an unnecessarily large lower bound imposed on the uncertainty in MISR observations at low light levels, and improves when this lower bound is removed; (2) Dark Water retrievals are able to distinguish between the spherical and non-spherical particles currently used for all MISR viewing geometries when the AOD exceeds 0.1 ; (3) the sensitivity of the MISR retrievals to aerosol non-sphericity varies in a complex way that depends on the sampling of the scattering phase
\end{abstract}

function and the contribution from multiple scattering; and (4) non-sphericity artifacts occur at those view-illumination geometries where dust aerosols are indistinguishable from certain types of cirrus particles. Based on these results, we suggest that interested parties use caution with the version 22 MISR Dark Water aerosol non-sphericity product in situations where cirrus may be present.

\section{Introduction}

Dust outbreaks are a common feature of the world's arid and semiarid regions. North African and East Asian dust storms have been documented for thousands of years, and these records have climate significance because dust storm frequency and severity affect soil moisture, air and surface temperatures, rainfall, and downwind air quality; and, at the same time, indicate past/current climate change (Sun et al., 2001; Shao and Dong, 2006). There are many welldocumented cases of dust from African sources being transported across the Atlantic, as well as dust from East Asian sources in Mongolia and China being transported across the Pacific and even around the globe (Eguchi et al., 2009; Uno et al., 2009; Yu et al., 2012). During long-range transport, dust particles can affect marine biochemistry by depositing iron in the ocean (Martin et al., 1991; Bopp et al., 2003; Mahowald et al., 2009), play a role in neutralizing acid rain (Wang et al., 2002), affect the life cycle of coral reefs (Shinn et al., 2000), and influence sea surface temperatures that impact hurricane formation and intensity (Dunion and Velden, 2004). In order to advance the assessment of climate change projections due to dust effects and forcing, one of the foremost needs is to develop new techniques for interpreting and 
merging the diverse information from satellite remote sensing, in situ and ground-based measurements, and model simulations (Sokolik et al., 2001; Formenti et al., 2011).

The development of new satellite sensors with improved observing capabilities for dust - notably the Multiangle Imaging SpectroRadiometer (MISR) (Kalashnikova and Kahn, 2008; Kahn et al., 2009b), the Meteosat Second Generation (MSG) Spinning Enhanced Visible and InfraRed Imager (SEVIRI) (Brindley and Ignatov, 2006; Brindley et al., 2012), the Ozone Monitoring Instrument (OMI), the Atmospheric Infrared Sounder (AIRS), the CloudAerosol Lidar with Orthogonal Polarization (CALIOP) (DeSouza-Machado et al., 2010), the Infrared Atmospheric Sounder Interferometer (IASI) (Klüser et al., 2012), and the POLarization and Directionality of the Earth's Reflectances (POLDER) (Peyridieu et al., 2013), as well as new satellite data-processing algorithms including "Deep Blue" for the Moderate Resolution Imaging Spectroradiometer (MODIS) (Hsu et al., 2004, 2006) and the Sea-viewing Wide Field-ofview Sensor (SeaWiFS) (Sayer et al., 2012) - has radically improved our ability to monitor and understand global dust emission and transport processes.

MISR's unique multiangular viewing geometry and imaging capability yield several geophysical products that can aid in understanding dust impacts on the climate system. MISR aerosol products, including total column aerosol optical depth (AOD) over bright land surfaces (Frank et al., 2007; Christopher et al., 2008, 2009; Koven and Fung, 2008; Kahn et al., 2009b), aerosol plume heights and associated wind vectors (Kahn et al., 2007b), and aerosol microphysical properties such as particle size AOD fractions and non-spherical AOD fraction (Kahn et al., 1997, 1998, 2001; Kalashnikova et al., 2005; Kalashnikova and Kahn, 2008), have been used to quantitatively constrain dust distributions and dynamics. The MISR AOD product, including retrieval performance in dusty regions, has been extensively validated (Martonchik et al., 2004; Kahn et al., 2005a, 2010).

The goal of this paper is to evaluate the sensitivity of the operational MISR version 22 (V22) Dark Water aerosol retrieval algorithm to aerosol non-sphericity as a function of viewing geometry. We present an assessment of the global sensitivities of multiangular observations to aerosol non-sphericity under the measurement uncertainties assumed based on the on-orbit performance of MISR multiangle intensity observations. Section 2 introduces the MISR instrument and the MISR non-spherical aerosol (dust) optical models, and describes the current, global, MISR non-spherical AOD fraction climatology. Section 3 discusses the MISR viewing geometry, MISR angular sampling over the ocean, and the effects of sunglint on this sampling. Section 4 introduces spherical and non-spherical optical models that are currently not included in the operational MISR look-up tables used in the Dark Water aerosol retrieval that could potentially be confused with the MISR dust models. Section 5 describes the overall MISR Dark Water retrieval approach.
In particular, the section introduces the $\chi^{2}$ tests for "goodness of fit" and shows how these are used by the MISR V22 Dark Water retrieval algorithm to determine the AOD and discriminate aerosol types. In Sect. 6, we investigate theoretical MISR sensitivities to differences between spherical and non-spherical aerosol models as functions of season and latitude in terms of $\chi^{2}$ values (absolute, spectral, geometric, and maximum deviation) and their associated thresholds used in V22 Dark Water retrievals. Specifically, we evaluate the distinguishability of the MISR medium-mode dust aerosol model from spherical and non-spherical (e.g., cirrus) particles, and discuss implications of the algorithm sensitivity to the non-sphericity artifacts observed in the MISR global non-spherical AOD fraction climatology. Section 6 presents conclusions, and discusses the range of applicability of the current, operational MISR non-sphericity product over the global oceans.

\section{MISR non-spherical AOD fraction product}

MISR (Diner et al., 1998) is one of five instruments on NASA's sun-synchronous, polar-orbiting Terra satellite, which crosses the Equator on its descending node at 10:30 LT. Terra was launched in December 1999 and MISR has been acquiring data since late February 2000. The MISR instrument consists of nine cameras with view angles of $\pm 70.5^{\circ}, \pm 60.0^{\circ}, \pm 45.6^{\circ}, \pm 26.1^{\circ}$, and $0^{\circ}$ (nadir), operating in four wavelengths centered at $446.4 \mathrm{~nm}$ (blue), $557.5 \mathrm{~nm}$ (green), $671.7 \mathrm{~nm}$ (red), and $866.4 \mathrm{~nm}$ (near infrared, or NIR). The camera configuration allows for a location to be imaged by all cameras and all wavelengths within a span of seven minutes. The map-projected spatial resolution of the red wavelength is $275 \mathrm{~m}$ in all nine cameras; the other wavelengths are averaged to $1.1 \mathrm{~km}$ resolution in all the cameras in the instrument's standard global observing mode except the nadir, which preserves the full $275 \mathrm{~m}$ resolution in all four wavelengths. The swath common to all nine cameras is $\sim 400 \mathrm{~km}$ wide, and global coverage is obtained every nine days at the Equator and more frequently at higher latitudes (Diner et al., 2002).

MISR operational (V22) Dark Water aerosol retrievals are reported for regions consisting of $16 \times 161.1 \mathrm{~km}$ image pixels, enclosing an area of $17.6 \mathrm{~km} \times 17.6 \mathrm{~km}$, or $310 \mathrm{~km}^{2}$. Total column AOD, and other information including aerosol Ångström exponent, size AOD fraction (small, medium, large), sphericity AOD fraction (spherical and nonspherical), and single-scattering albedo (SSA), as well as additional parameters and diagnostics are contained in the MISR Level 2 Aerosol (L2AS) product, which is archived at the NASA Langley Atmospheric Sciences Data Center (http://eosweb.larc.nasa.gov/PRODOCS/misr/table_misr. html). The V22 MISR aerosol retrieval algorithm uses a set of 74 prescribed aerosol mixtures, containing up to three "pure particles" represented by log-normal size distributions 

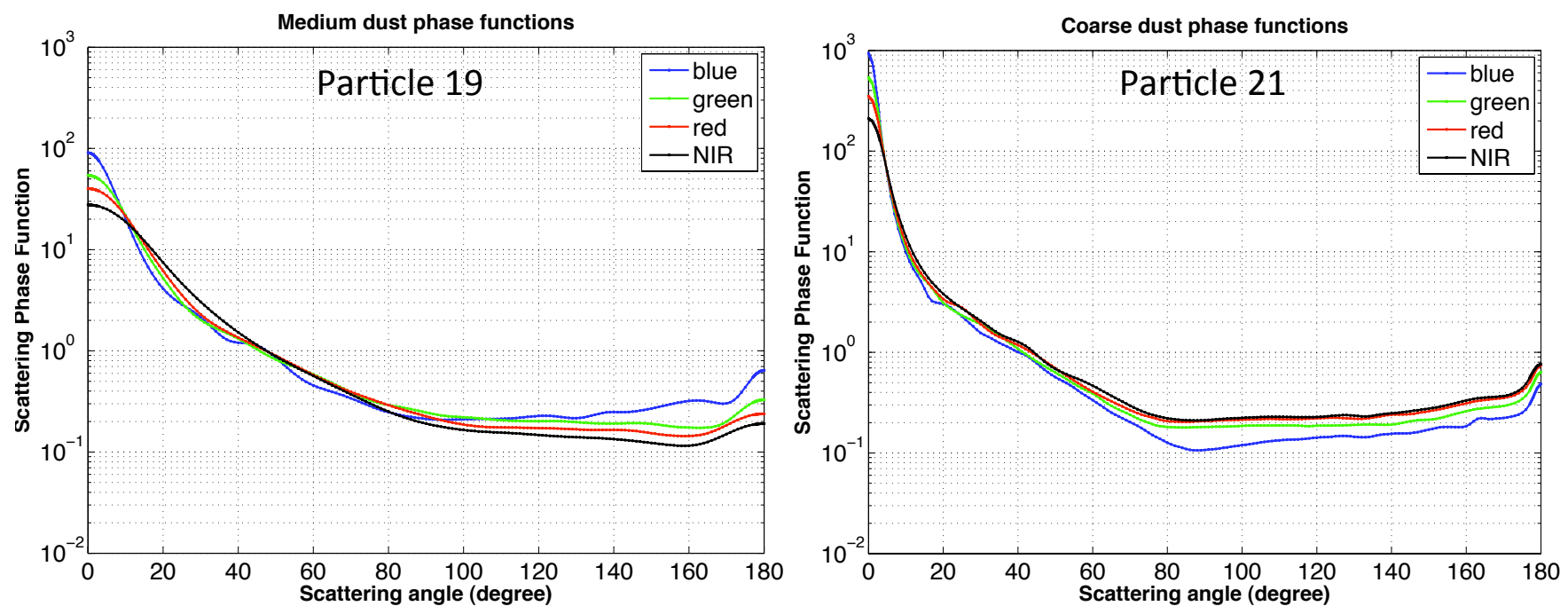

Fig. 1. The scattering phase functions of MISR Particle 19 (medium dust) and MISR Particle 21 (coarse dust) at the four MISR wavelengths: $447 \mathrm{~nm}$ (blue), $558 \mathrm{~nm}$ (green), $672 \mathrm{~nm}$ (red), and $866 \mathrm{~nm}$ (NIR). Optical properties of these models are listed in Table 1.

having a specific number-weighted median radius, width, and fixed indices of refraction. Twenty-four of the aerosol mixtures in the V22 set include non-spherical (dust) components (Martonchik et al., 2009; Kahn et al., 2010). Among these dusty mixtures, 12 mixtures contain a medium-sized dust particle mixed with small and large, non-absorbing, spherical particles; 8 mixtures contain bimodal dust distributions (medium and coarse dust components) mixed with small, non-absorbing, spherical particles; and 4 are solely dust, containing only the 2 dust particles. The particle information is stored in a look-up table (LUT) known as the Simulated MISR Ancillary Radiative Transfer (SMART) dataset.

The two MISR dust particle models were developed based on morphological and mineralogical properties of natural dust collected in the atmosphere as described in Kalashnikova and Sokolik (2004) and Kalashnikova et al. (2005), and were implemented in MISR operational aerosol retrievals beginning with version 16 as MISR Particle 19 (medium dust) and MISR Particle 21 (coarse dust) (see, for example, Kahn et al., 2010, Table 2). Optical properties for the two MISR dust models are summarized in Table 1, and the scattering phase functions for the two models at the four MISR wavelengths are shown in Fig. 1. The medium dust mode consists of weakly absorbing, grain-shaped particles (number-weighted median radius $=0.5 \mu \mathrm{m}$, and the standard deviation of the log-normal distribution is given by the natural logarithm of $\sigma=1.5$ ). The optical properties for this mode at three MISR wavelengths (green, red, and NIR) were developed as described by Kalashnikova et al. (2005). The coarse dust mode (number-weighted median radius $=1 \mu \mathrm{m}$ and $\sigma=2.0$ ), which at the time could not be modeled with the discrete dipole approximation (DDA) technique due to its size parameter limitation (Draine and Flatau, 1994), was instead modeled using the T-matrix technique (Mishchenko et al., 1996) assuming uniformly mixed oblate and prolate spheroids with aspect ratios ranging from 1.2 to 3 , and the same refractive indices as the medium dust mode. The maximum diameter used in the calculation of the scattering phase function for the medium dust mode was $2 \mu \mathrm{m}$, and for the coarse dust mode the maximum diameter was $8 \mu \mathrm{m}$ (i.e., both size distributions were truncated due to optical model limitations; see Kalashnikova et al., 2005). The MISR V22 Dark Water aerosol retrieval relies primarily on observations from the two longest MISR wavelengths (672 and $866 \mathrm{~nm}$ ), where the water surface is dark (Martonchik et al., 2002). However, when the AOD exceeds 0.5 the $588 \mathrm{~nm}$ (green) wavelength begins to be utilized, and then when the AOD exceeds 0.75 , all four wavelengths are used in the Dark Water aerosol retrieval (Diner et al., 2005). The earlier V16 of the algorithm did not utilize the blue wavelength, so the accuracy of the dust optical models at this wavelength was not considered important. Therefore, spheroidal models with the same size distributions described above, and a single fixed aspect ratio of 1.5 , were used to model the optics of both dust modes at $446 \mathrm{~nm}$ (blue) using the T-matrix technique. Figure 1 clearly demonstrates the differences in the shape of the scattering phase functions for both dust models for the MISR blue wavelength relative to the phase functions at the longer wavelengths, which is due to the difference in the optical modeling technique.

Figure 2 shows an example of the MISR Dark Water algorithm performance over a dusty region of the Atlantic Ocean before and after the new dust models were implemented. The inset globe indicates the approximate location of the MISR observations. The true color browse image from the MISR Df $\left(+70.5^{\circ}\right)$ camera is shown on the far left for context. The image was acquired on 6 February 2004 at 12:15 UTC (MISR Orbit 22006, Path 210). The Cape Verde archipelago 
Table 1. Optical properties of MISR dust components.

\begin{tabular}{ccccccccccc}
\hline Size & Shape & $n_{\mathrm{r}}$ & $\begin{array}{c}\text { Blue } \\
n_{\mathrm{i}} \times 10^{3}\end{array}$ & $\begin{array}{c}\text { Green } \\
n_{\mathrm{i}} \times 10^{3}\end{array}$ & $\begin{array}{c}\text { Red } \\
n_{\mathrm{i}} \times 10^{3}\end{array}$ & $\begin{array}{c}\text { NIR } \\
n_{\mathrm{i}} \times 10^{3}\end{array}$ & $\begin{array}{c}\text { Blue } \\
\omega_{0}\end{array}$ & $\begin{array}{c}\text { Green } \\
\omega_{0}\end{array}$ & $\begin{array}{c}\text { Red } \\
\omega_{0}\end{array}$ & $\begin{array}{c}\text { NIR } \\
\omega_{0}\end{array}$ \\
\hline Medium & Grains & 1.51 & 4.112 & 2.111 & 0.652 & 0.472 & 0.919 & 0.977 & 0.994 & 0.996 \\
Coarse & Spheroids & 1.51 & 4.112 & 2.111 & 0.652 & 0.472 & 0.810 & 0.902 & 0.970 & 0.983 \\
\hline
\end{tabular}

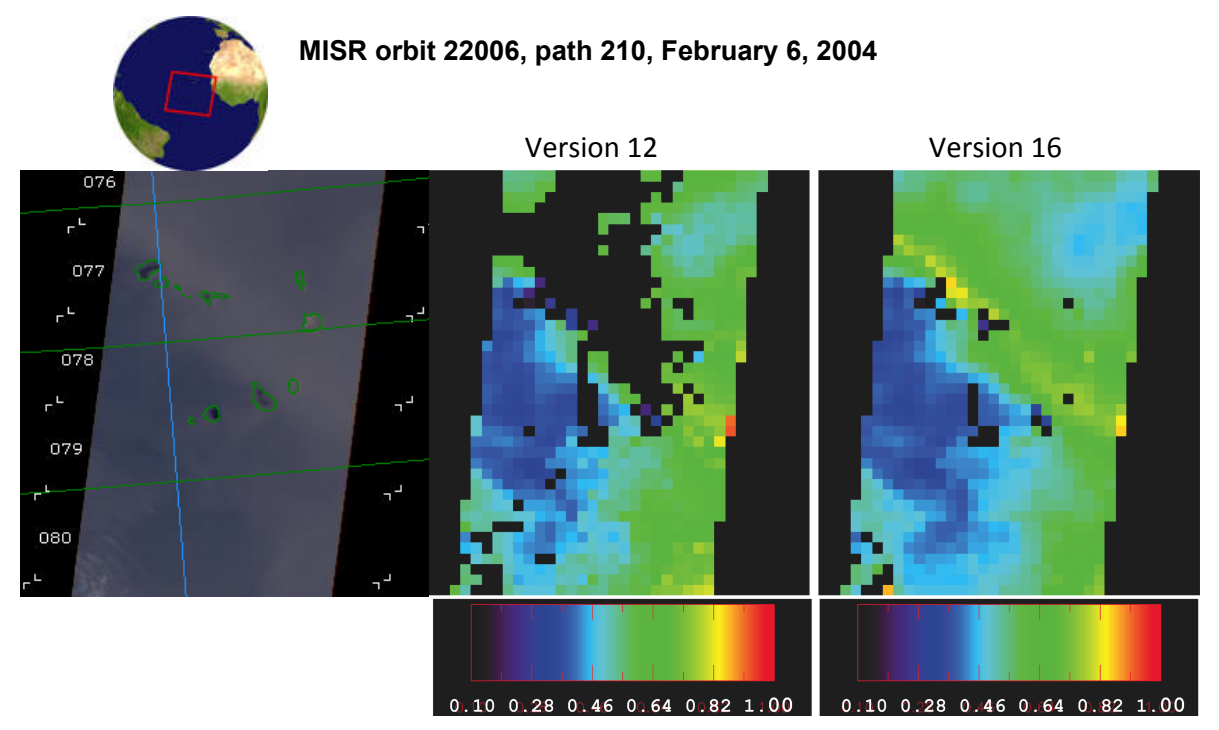

Fig. 2. Example MISR scene of dust over the Atlantic, Orbit 22006, Path 210, 6 February 2004, 12:15 UTC. Left: true color browse image from the MISR Df $\left(+70.5^{\circ}\right)$ camera. Center: version 12 operational MISR AOD retrievals for this region before the incorporation of the current non-spherical (dust) models. Right: version 16 operational MISR AOD retrievals after the inclusion of current dust models.

is located near the center of the image, and the brownish haze over the entire scene indicates the presence of a significant amount of atmospheric dust. The center panel of Fig. 2 shows the green-wavelength AOD retrieved by the MISR V12 Dark Water algorithm, and the right-hand panel shows the AOD retrieved by the V16 algorithm, which incorporates the dust models described above. The blank portion in the upper part of the center panel corresponds to regions where the V12 algorithm was unable to find any aerosol model that adequately fits the MISR observations. This region is filled in the V16 image because the new dust models provided adequate fits, resulting in successful retrievals.

Globally, MISR AOD coverage improved significantly in V16 and later versions of the aerosol product, and the AOD retrievals have since been validated by extensive intercomparisons with ground-based AERONET sunphotometer observations (Kahn et al., 2005a, 2010; Kalashnikova and Kahn, 2008) and by field experiment closure studies (Kahn et al., 2009b). Figure 3 shows the monthly MISR global climatology of mid-visible AOD retrieved over dark water from V22 of the algorithm averaged over the 12 years of the mission. The dominant features are aerosols transported from nearby source regions off the coast of Africa that are primarily biomass burning or mineral dust depending on the season and location, dust in the Indian Ocean from the Arabian Peninsula driven by the monsoon circulation, and dust transport across the northern Pacific Ocean in the spring. Comparisons of MISR global AOD with other satellite datasets over water are consistent and show good overall agreement (Kinne et al., 2006; Kahn et al., 2009a; Zhang and Reid, 2010; Shi et al., 2011).

Current MISR V22 Dark Water retrievals predominantly select MISR Particle 19 (medium dust) as the best fitting (lowest residual) aerosol model in dusty regions. The insitu observations obtained during the Saharan Mineral Dust Experiment part 1 (SAMUM-1) (Schladits et al., 2009) further demonstrated the representativeness of the MISR dust optical models as compared to dust optical properties measured in the field (e.g., for Saharan dust). However, an unexpected consequence of introducing the dust optical models into the MISR aerosol retrieval algorithm is that significant fractions of non-spherical particles appear around the globe in locations where mineral dust is not expected to be observed. The overall distribution, characteristics, and behavior of the non-spherical AOD fraction (i.e., the retrieved nonspherical fraction weighted by the total AOD) are best seen on global monthly gridded aerosol property maps that reveal spatial and temporal patterns that are difficult to discern 

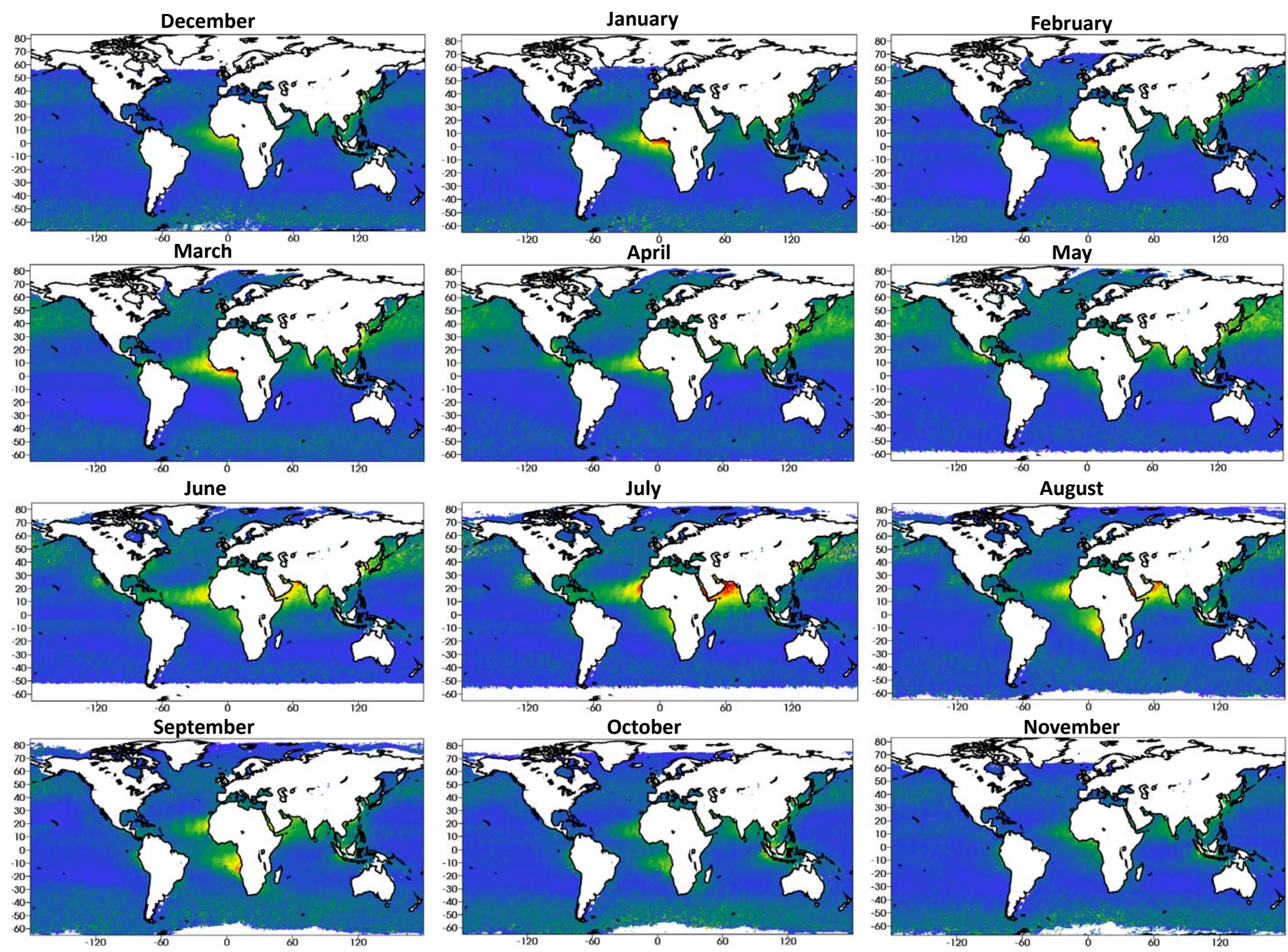

MISR AOD (2000-2012)

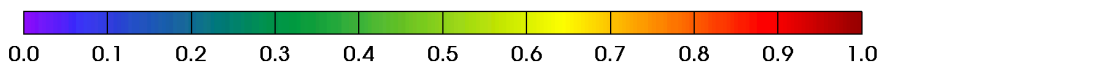

Fig. 3. Monthly climatology of MISR V22 green-wavelength AOD retrieved over dark water, averaged over 12 years: March $2000-$ February 2012.

when viewing individual level 2 (swath) retrievals or seasonal maps. Figure 4 shows the MISR monthly climatology of non-spherical AOD fraction for AOD greater than 0.15, corresponding to the minimum AOD threshold suggested in previous sensitivity and validation studies (Kahn et al., 2001; Kalashnikova and Kahn, 2006; Kahn et al., 2010), and averaged over 12 years of observations (March 2000February 2012) gridded at $0.5^{\circ}$ resolution. These maps show large scale zonal bands of enhanced non-spherical AOD fraction in both the Northern and Southern Hemispheres. Superimposed on these bands are the expected dust transport regions dominated by equatorial Africa, the Middle East, and eastern Asia, with contributions from southern South America, southern Africa, and Australia (see, for example, Mahowald et al., 2009, Fig. 1b). The Southern Hemisphere band of enhanced non-sphericity is more clearly defined and shifts noticeably north and south over time, and similar behavior can be seen for the band in the Northern Hemisphere as well. Note that these artifacts do not appear to affect the retrieval of overall AOD shown in Fig. 3. It is also important to note that maps of the non-spherical AOD (AOD $\times$ non-spherical fraction) do not show these artifacts either as the non-spherical AOD tends to be dominated by locations with high AOD.

One hypothesis for the appearance of these bands is that they occur in regions with low AOD where the MISR Dark Water algorithm has reduced sensitivity to aerosol properties. Previous MISR studies (Kahn et al., 2001; Kalashnikova and Kahn, 2006) demonstrated that over dark water the MISR aerosol retrieval is sensitive to differences in the angularspectral signals of medium-mode dust shapes when the midvisible AOD is larger than about 0.15 for components contributing $15-20 \%$ or more to the total AOD. This hypothesis is tested in Fig. 5, which shows MISR non-spherical AOD fraction for the month of October averaged over 

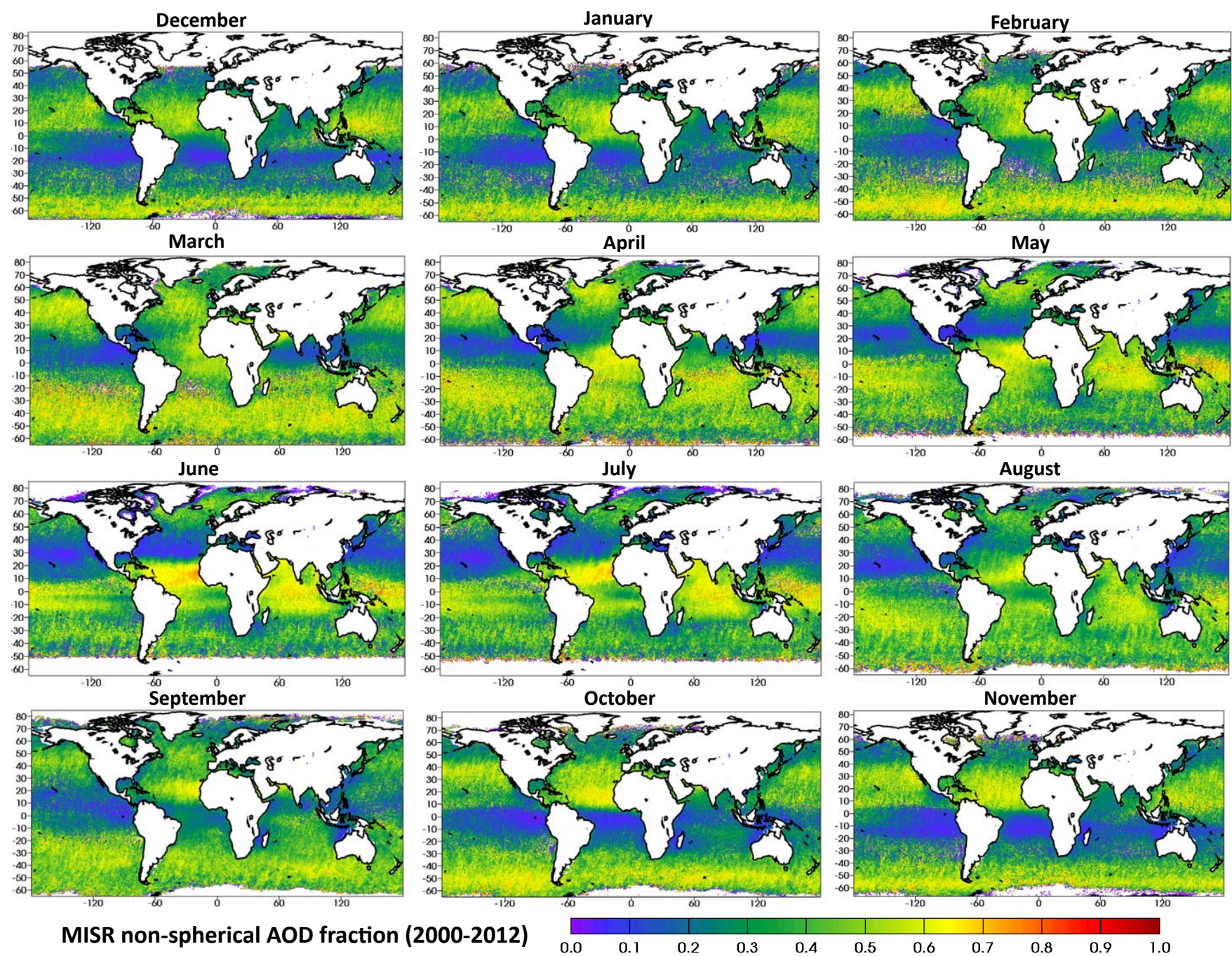

Fig. 4. Monthly climatology of MISR V22 non-spherical AOD fraction over dark water, averaged over 12 years: March $2000-$ February 2012.

$12 \mathrm{yr}$ and subsetted by different ranges of minimum AOD: $\mathrm{AOD}>0.15, \mathrm{AOD}>0.30, \mathrm{AOD}>0.40$, and $\mathrm{AOD}>0.50$. Notice that, although the coverage is reduced, the bands of enhanced non-sphericity still appear regardless of the range of AODs used. The band of enhanced non-sphericity in the Southern Ocean, for example, is evident in all four panels, indicating that reduced retrieval sensitivity at low AOD is not the cause of the non-sphericity bands.

Other potential reasons for these non-sphericity bands, which will be explored in greater detail below, are as follows: (1) artifacts caused by a reduced range of scattering angles observed by the MISR cameras in certain latitudes and seasons, (2) reduced sensitivity to the difference between spherical and non-spherical particles for certain MISR viewing geometries, and (3) unscreened cirrus retrieved as nonspherical dust by the Dark Water algorithm due to the lack of cirrus models in the operational MISR SMART dataset.
3 Enhanced bands of particle non-sphericity and MISR angular sampling over ocean

In this section we investigate whether the seasonally variable bands of enhanced non-sphericity apparent in Fig. 4 are related to the range of scattering angles observed by the MISR instrument. In particular, we investigate how the actual viewing geometry of the MISR cameras changes over the global oceans and the effect this changing geometry has on the information used by the operational V22 Dark Water aerosol retrieval algorithm. For this discussion, it is convenient to consider the scattering angle, $\Theta$, because it is the variable used to represent scattering phase functions, such as those shown in Fig. 1. The scattering angle is related to the planeparallel viewing geometry of a satellite instrument through the expression

$\cos \Theta=-\mu \mu_{0}+\nu \nu_{0} \cos (\Delta \phi)$, 
(a) $A O D>0.15$

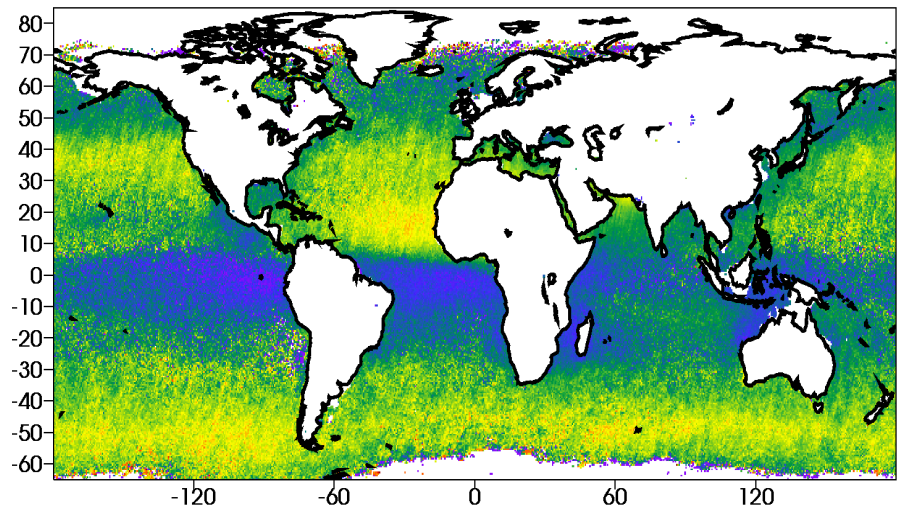

(c) $A O D>0.4$

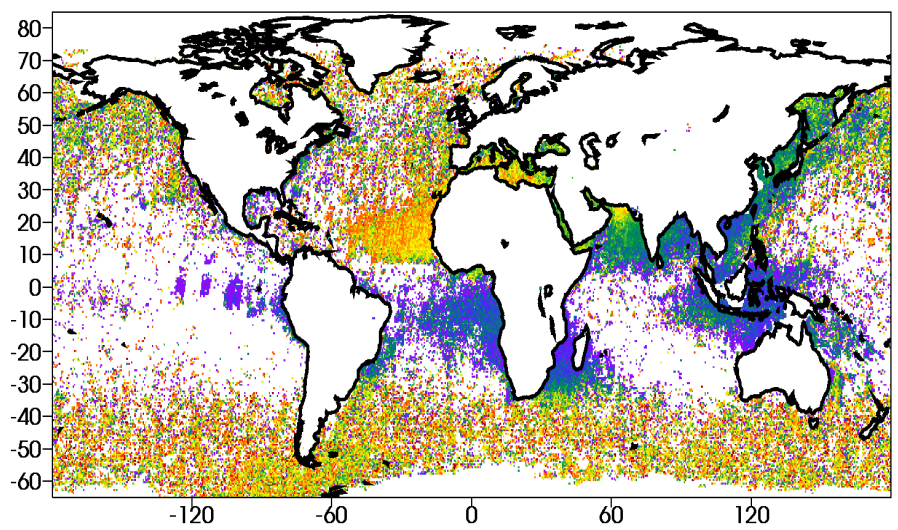

(b) AOD $>0.3$

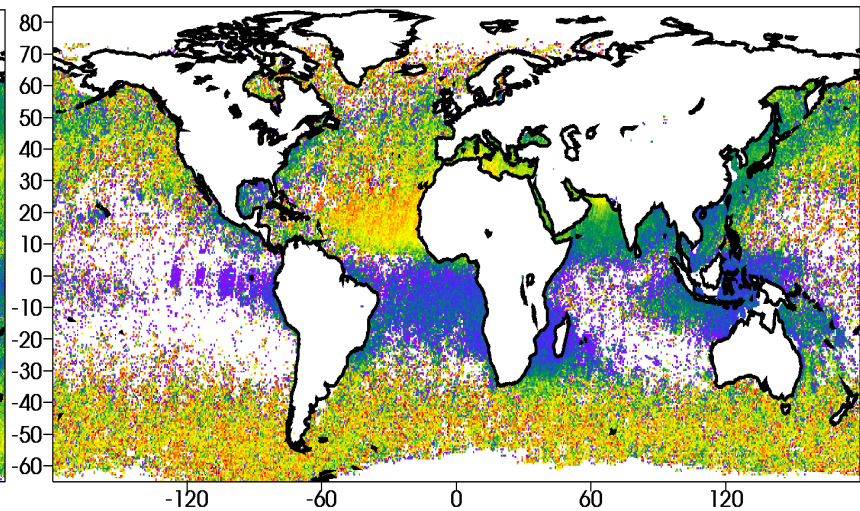

(d) AOD $>0.5$

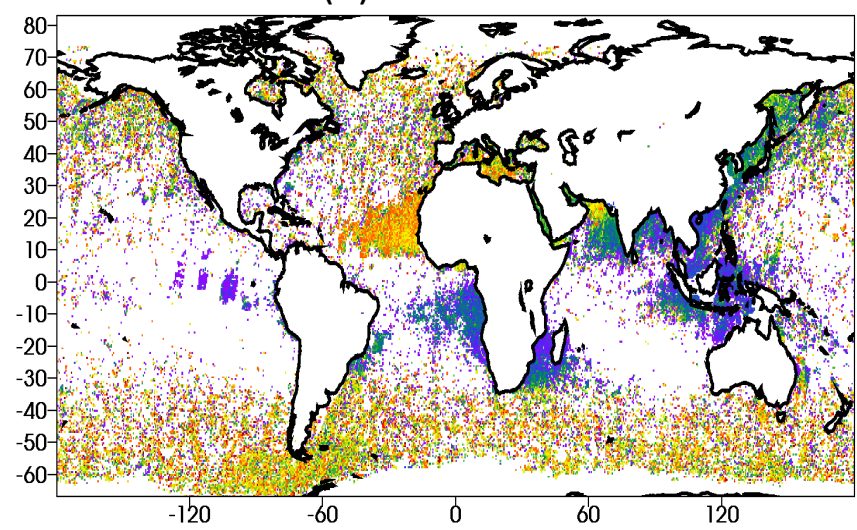

MISR non-spherical AOD fraction 0.0
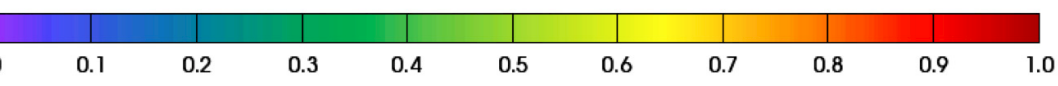

Fig. 5. Climatology of MISR V22 non-spherical AOD fraction for the month of October over dark water for different AOD ranges: (a) $\mathrm{AOD}>0.15$, (b) $\mathrm{AOD}>0.3$, (c) $\mathrm{AOD}>0.4$, (d) $\mathrm{AOD}>0.5$. This figure illustrates that the bands of enhanced non-sphericity are not simply due to reduced algorithm sensitivity at low AOD.

where $\mu$ and $v$ are the cosine and sine of the view zenith angle, respectively; $\mu_{0}$ and $\nu_{0}$ are the cosine and sine of the solar zenith angle, respectively; and $\Delta \phi$ is the relative azimuth angle, which is the difference between the view azimuth and solar azimuth angles (Davies, 1980).

Prelaunch studies indicated that the sensitivity of MISR Dark Water aerosol retrievals to particle properties would be diminished at latitudes where the range of scattering angles sampled by the nine MISR cameras did not adequately capture the important features of the particle scattering phase function (Kahn et al., 1997, 1998, 2001). Later sensitivity studies, focusing on the ability of the MISR Dark Water aerosol retrieval algorithm to distinguish spherical from nonspherical particles, however, only investigated algorithm performance for mid-latitude viewing geometries in an attempt to represent the average overall performance of a global aerosol retrieval (Kalashnikova and Kahn, 2006; Pierce et al., 2010).
As Fig. 6 demonstrates the range of scattering angles, $\Delta \Theta$ $=\Theta_{\max }-\Theta_{\min }$, seen by the full set of nine MISR cameras actually varies significantly with latitude, season, and across the MISR swath (Kahn et al., 1997). The horizontal axis on the figure corresponds to time, with MISR swaths overlaid every 16 days, which is the repeat period of the Terra satellite. The vertical axis depicts the variability with latitude, so the overall figure is similar to a classic Hovmöller diagram (Hovmöller, 1949). An important difference is that Fig. 6 also captures the variability across the $\sim 400 \mathrm{~km}$ MISR swath, which would be lost if a longitudinal average had been performed. Because the Terra satellite is sun-synchronous, the structure of an individual swath does not vary with longitude. As the north-south position of the sun varies less than $4^{\circ}$ over the 16-day time period represented by each individual swath in Fig. 6, the swaths themselves effectively depict the average scattering angle range for each time period. Note that MISR aerosol retrievals are not performed when 


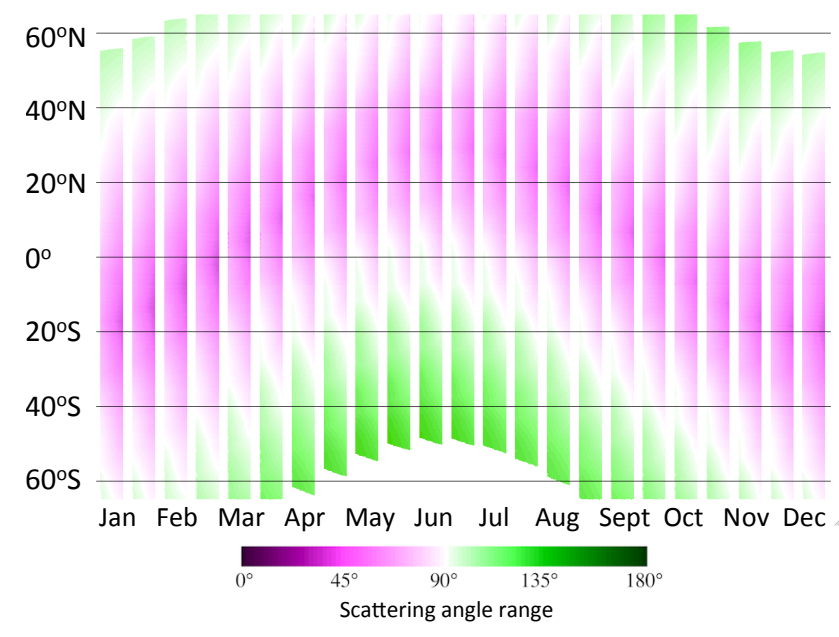

Fig. 6. Scattering angle range $\left(\Theta_{\max }-\Theta_{\min }\right)$ sampled considering all nine MISR cameras, displayed as a function of latitude and time of year.

the cosine of the solar zenith angle is less than 0.20 (i.e., when the solar zenith angle exceeds $78.46^{\circ}$ ), which accounts for the blank areas in the bottom center of the figure, as well as in the upper left and upper right. As expected, the scattering angle range, $\Delta \Theta$, sampled by all nine MISR cameras is greatest at high latitudes, reaching a maximum around $140^{\circ}$, and is smaller at low latitudes, with the smallest range near the latitude of the subsolar point, where the scattering angle range can be as small as $40^{\circ}$ (Kahn et al., 1997, 2001).

Figure 6 represents the situation when all nine MISR cameras are considered, which is the case for most aerosol retrievals performed over land. Over ocean, the situation is complicated by the effects of sunglint, which is the specular reflection of sunlight from the ocean surface (Ottaviani et al., 2008). Cameras sampling angles within $40^{\circ}$ of the specular direction are not used in MISR Dark Water retrievals to minimize the effects of sunglint (Kahn et al., 2007a). Figure 7a is similar to Fig. 6, but excludes cameras potentially affected by sunglint from the calculation of the scattering angle range, $\Delta \Theta$. Notice that at high latitudes the range of scattering angles changes from about $140^{\circ}$ in Fig. 6 to as small as $40^{\circ}$ in Fig. 7a. Figure 7b indicates the maximum number of cameras that are actually available to be used in the MISR Dark Water aerosol retrieval, which can range from four to nine. The complex structure shown in the figure is caused by different cameras being excluded at different latitude ranges as they move into and out of the $40^{\circ}$ sunglint cone. If the number of valid cameras is less than four, no retrievals are attempted over water. The maximum number of valid cameras typically corresponds to the maximum scattering angle range, except near the subsolar point where the MISR cameras only sample a relatively small portion of scattering angle space.

The approach used to generate Figs. 6 and 7 can also be used to depict the non-spherical AOD fraction climatology over the global oceans, as shown in Fig. 8. The temporal evolution of the bands of enhanced particle non-sphericity is clearly evident in this figure, which can be compared directly with Fig. 6. This comparison shows that the bands move north and south over time in a manner similar to the patterns in the scattering angle range. Exceptions to this behavior are the enhanced non-spherical AOD fraction from 10 to $20^{\circ} \mathrm{N}$ from April through August that corresponds to the transatlantic transport of Saharan dust (Kaufman et al., 2005; Kalashnikova and Kahn, 2008). Another region of enhanced non-spherical AOD fraction, apparent between 35 and $50^{\circ} \mathrm{N}$ from February to May, corresponds to the trans-Pacific transport of Asian dust (Yu et al., 2012). This region is more difficult to make out because it overlaps with the temporally varying band of enhanced particle non-sphericity in the Northern Hemisphere.

Close examination of this figure and the previous two, however, reveals that the location of the bands of enhanced non-sphericity do not precisely correspond to the location of the smallest number of valid MISR cameras or the smallest sampling of the scattering angle range. In fact, enhanced nonsphericity is apparent at latitudes where the sampling of the scattering angle range is largest, and on the left-hand side of the MISR swath where the number of valid cameras is greatest. This indicates that the non-sphericity bands are not a simple function of the reduction in the sampling of the scattering angle range, or a reduction in the number of available cameras, as might be expected on theoretical grounds alone (Kahn et al., 1997, 1998, 2001).

In Fig. 9 we further investigate the possibility that the bands of enhanced non-sphericity are due to a reduction of the sensitivity of the MISR Dark Water aerosol retrieval algorithm when the AOD is small. While Fig. 8 shows the MISR non-spherical AOD fraction climatology for all AODs, Fig. 9a shows the climatology only for AOD $>0.15$, and Fig. $9 \mathrm{~b}$ shows the climatology only for AOD $>0.40$. As the minimum AOD increases, the spatial extent and the absolute magnitude of the non-spherical fraction both increase. The speckled appearance of the AOD $>0.40$ figure is due to decreased sampling in this optical depth range over much of the global oceans away from significant aerosol sources. The regions of true dust transport across the Atlantic and Pacific, discussed above, also become more apparent because they are dominated by high aerosol loading and therefore high AOD. Taken together, Figs. 8 and 9 demonstrate that the enhanced non-sphericity artifacts in the MISR V22 global ocean aerosol climatology are not simply related to the range of scattering angles sampled by the MISR cameras or reduced algorithm sensitivity at low AOD. This suggests that the apparent non-sphericity artifacts are due to the presence of aerosols with optical properties that the V22 Dark Water algorithm cannot distinguish from the dust models in the existing MISR SMART at certain view-illumination geometries. 
(a)

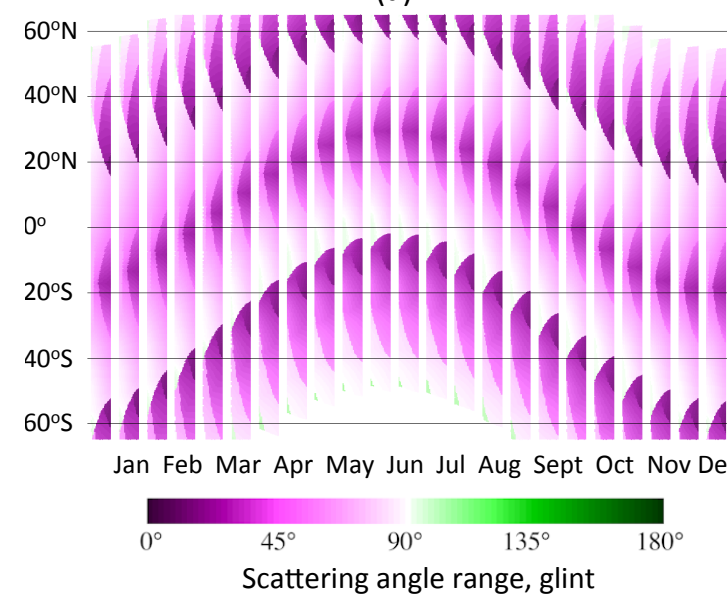

(b)

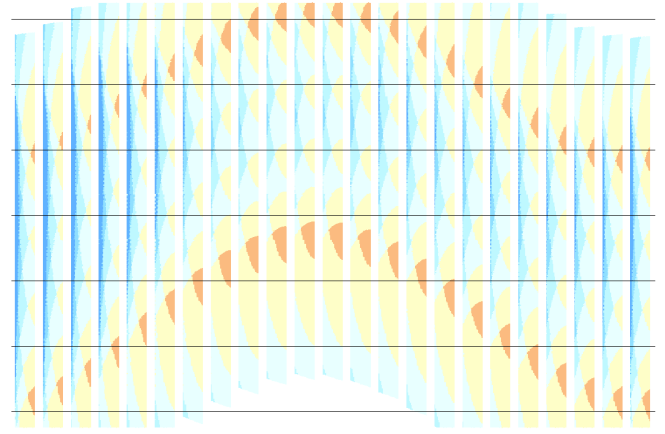

Jan Feb Mar Apr May Jun Jul Aug Sept Oct Nov Dec

$\begin{array}{llllll}4 & 5 & 6 & 7 & 8 & 9\end{array}$

Number of valid cameras

Fig. 7. (a) Scattering angle range $\left(\Theta_{\max }-\Theta_{\min }\right)$ excluding cameras within $40^{\circ}$ of the specular direction that could potentially be affected by sunglint, presented in the same manner as Fig. 6. (b) Maximum number of MISR cameras available for dark water retrievals accounting for excluded cameras. The minimum number of cameras used in MISR Dark Water aerosol retrievals is four.

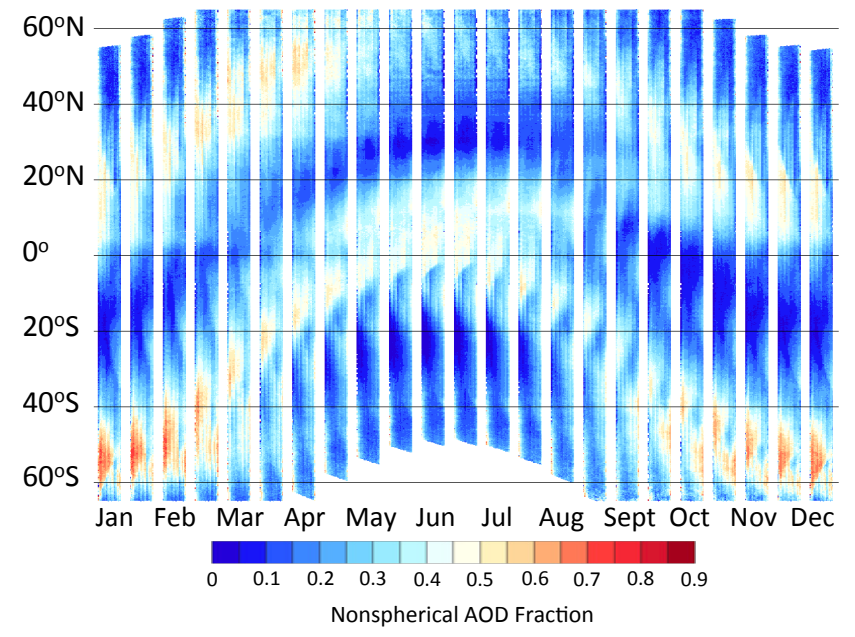

Fig. 8. Climatology of MISR non-spherical AOD fraction over ocean for all AODs averaged over 12 years (March 2000February 2012) presented in the same manner as Fig. 6.

\section{Additional aerosol optical models}

In order to better understand the role of aerosol models not included in the operational MISR SMART, we begin by examining how the MISR cameras sample the scattering phase function of different particles. A significant strength of the MISR observing approach is that each camera typically observes a different scattering angle, and thus a different part of the phase function (Diner et al., 2005). If the camera geometry is such that the scattering angles sampled are primarily backscattering $\left(\Theta>90^{\circ}\right)$, the ability of the retrieval algorithm to distinguish different particles is likely to be different than if the scattering angles lie in the more forward scattering $\left(\Theta<90^{\circ}\right)$ direction. One hypothesis we wish to test is whether or not reduced sensitivity to the difference between spherical and non-spherical particles at certain viewing geometries is the explanation for the seasonally varying nonsphericity artifacts in Fig. 8. In other words, naturally occurring spherical aerosols missing from the MISR SMART could potentially be retrieved by the MISR V22 Dark Water algorithm instead of non-spherical aerosols due to similarities in their scattering phase functions. A second hypothesis examined in this section is whether unscreened cirrus is being retrieved as non-spherical dust due to the lack of any cirrus models in the SMART dataset.

Figure 10 shows the latitudinal and seasonal distribution of the MISR minimum and maximum scattering angle, $\Theta_{\min }$ and $\Theta_{\max }$, over the global oceans accounting for the elimination of particular cameras due to potential sunglint contamination, as described above. Comparison of Fig. 10 with Fig. 7a shows that the range of scattering angles, $\Delta \Theta$, alone does not provide a complete picture of how the phase function is sampled. For example, the range of scattering angles sampled in the viewing geometry of Asian dust transport, indicated by the black box in Fig. 10, is similar to the range of scattering angles sampled in the viewing geometry of Saharan dust transport, indicated by the yellow box. However, the angles sampled in the Asian dust transport region are from about 130 to about $165^{\circ}$, while the angles sampled in the Saharan dust transport region are only from 110 to about $135^{\circ}$. The effect this has on particle discrimination is illustrated in greater detail in Figs. 11 and 12.

In Fig. 11, Particle 19 (medium dust) was selected as the reference model from the existing MISR SMART. This is due to the fact that, globally, Particle 19 is selected as the non-spherical aerosol component by the V22 Dark Water retrieval far more often than Particle 21 (coarse dust). The goal is to test whether a spherical particle can be found that 

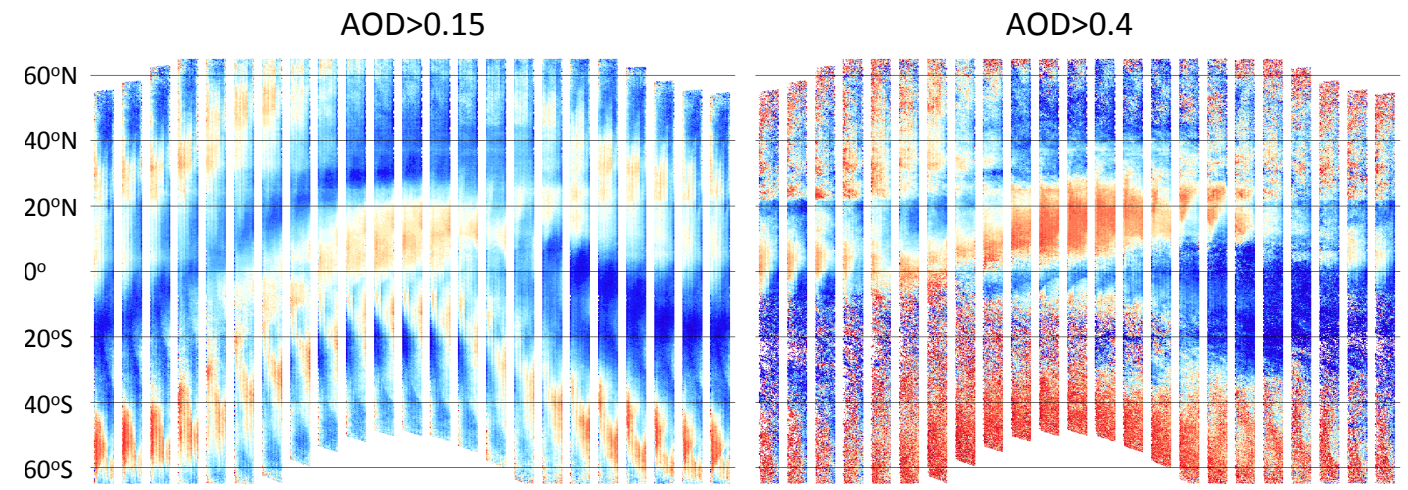

Jan Feb Mar Apr May Jun Jul Aug Sept Oct Nov Dec Jan Feb Mar Apr May Jun Jul Aug Sept Oct Nov Dec

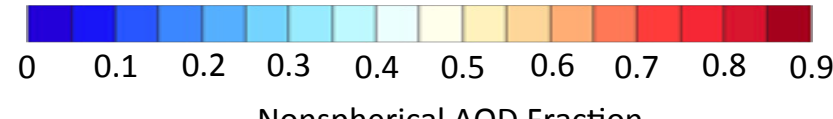

Fig. 9. Same as Fig. 8 but for different ranges of AOD: (a) AOD $>0.15$ and (b) AOD $>0.4$.

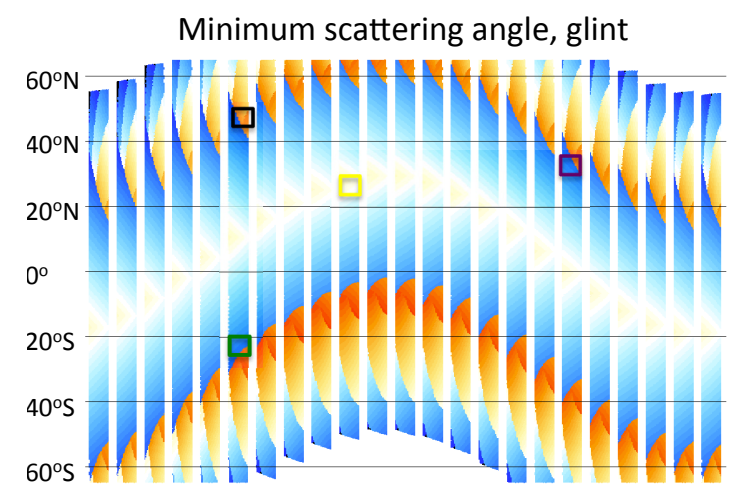

Jan Feb Mar Apr May Jun Jul Aug Sept Oct Nov Dec

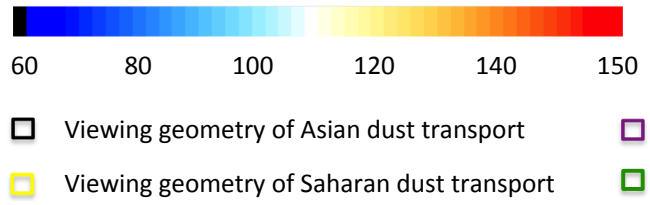

Maximum scattering angle, glint

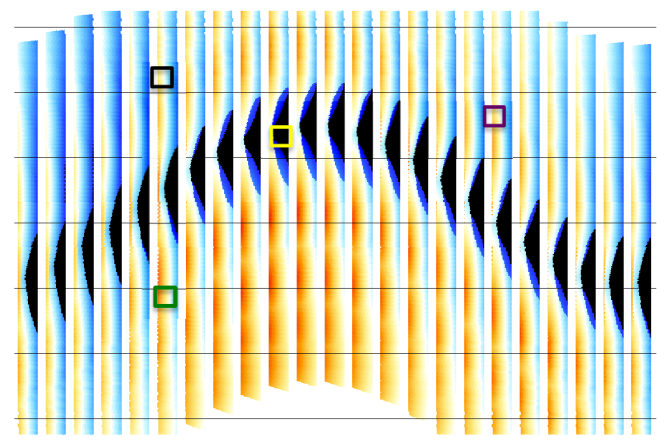

Jan Feb Mar Apr May Jun Jul Aug Sept Oct Nov Dec

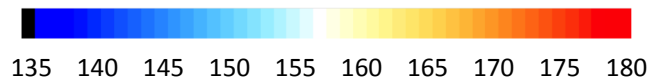

Viewing geometry of the Northern hemisphere band

Viewing geometry of the Southern hemisphere band

Fig. 10. (a) Minimum scattering angle $\left(\Theta_{\min }\right)$ used in the MISR Dark Water retrieval algorithm excluding cameras within $40^{\circ}$ of the specular direction that could potentially be affected by sunglint, presented in the same manner as Fig. 6. (b) Maximum scattering angle $\left(\Theta_{\text {max }}\right)$ used in the MISR Dark Water aerosol retrieval algorithm. Colored boxes indicate latitudes and times for viewing geometries of interest.

has a phase function similar enough to the phase function of the reference dust model so as to be indistinguishable given the scattering angles actually observed by the MISR cameras, particularly for the geometries in the bands of enhanced particle non-sphericity. In this case, we are exploring the hypothesis that the "true" aerosols are spherical, but are missing from the MISR SMART and the dust model is the closest analogue. Similar to what was done in previous studies (Kahn et al., 1997; Kalashnikova et al., 2005; Kalashnikova and Kahn, 2006), one of the two spherical particles selected for comparison was a size-equivalent sphere (number-weighted median radius of $0.5 \mu \mathrm{m}, \sigma=1.5$, maximum diameter $2 \mu \mathrm{m}$ ). Because these earlier studies showed that the phase function of a size-equivalent spherical particle has a dramatically different shape in the backscattering direction $\left(\Theta>130^{\circ}\right)$, a second spherical particle was selected to better match the Particle 19 scattering phase function in the backscattering direction with similar behavior in the MISR red and NIR wavelengths. We call this particle a "backscatter-equivalent sphere." The backscatter-equivalent sphere has a number-weighted median radius of $0.205 \mu \mathrm{m}$ and $\sigma=2.71$. Note that the backscatter-equivalent sphere 

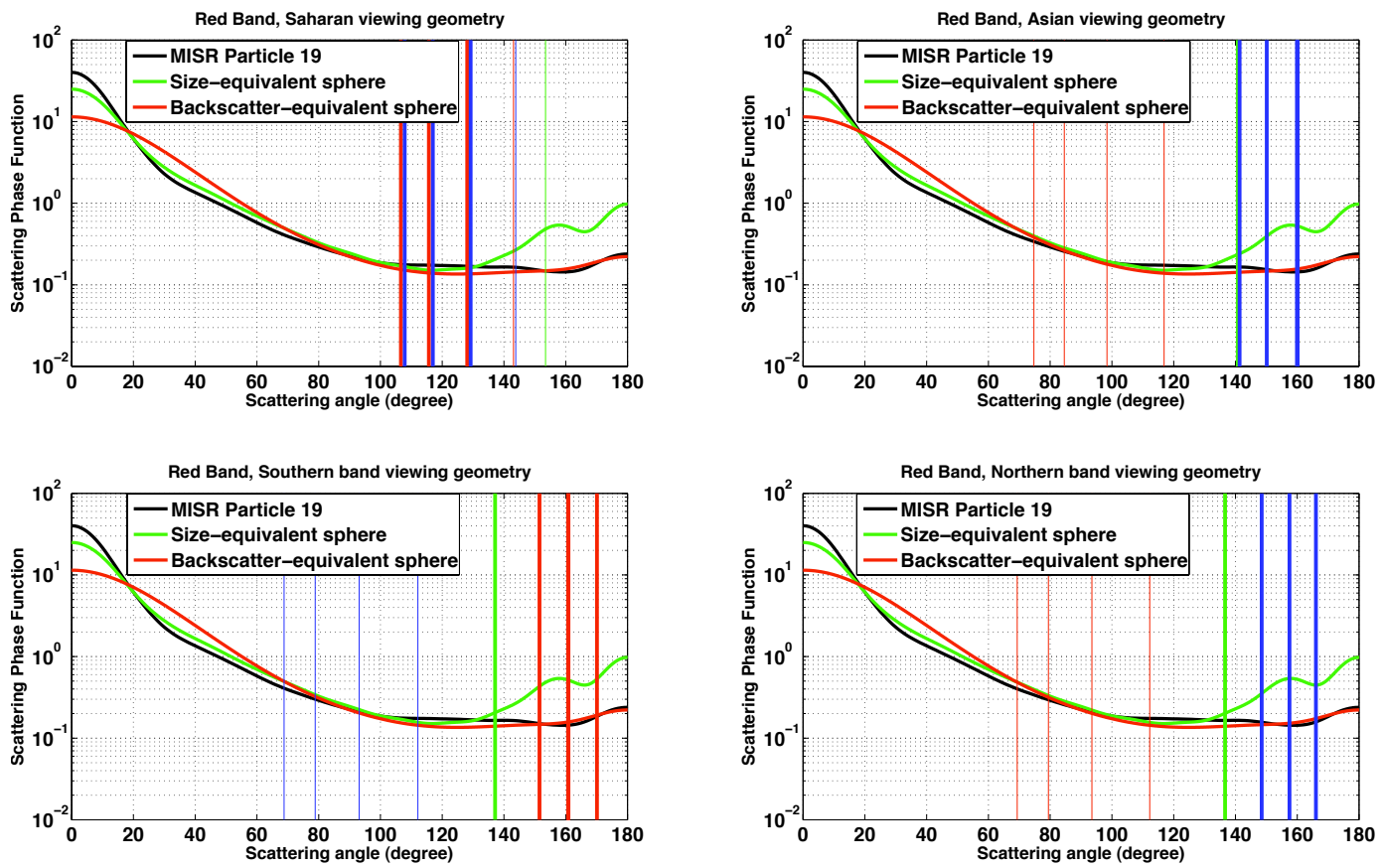

Fig. 11. Scattering phase functions for the MISR red wavelength for MISR Particle 19 (medium dust) compared with two additional spherical particles, a size-equivalent sphere and a backscattering-equivalent sphere. Different panels correspond to different viewing geometries indicated by the boxes in Fig. 10. (a) Saharan dust transport, (b) Asian dust transport, (c) Southern Hemisphere enhanced non-sphericity band, and (d) Northern Hemisphere enhanced non-sphericity band. Vertical lines indicate scattering angles observed by different MISR cameras with forward-looking cameras shown in red, aftward-looking cameras shown in blue, and the nadir camera shown in green. Thin lines indicate cameras with glitter angles within $40^{\circ}$ of the specular direction not used in the the MISR Dark Water retrieval.

has a much smaller number-weighted median radius than the size-equivalent sphere.

Figure 11 plots the phase functions for these three particles for the MISR red wavelength for the four different viewing geometries indicated by the boxes in Fig. 10. Vertical lines in each of the panels indicate the scattering angles observed by the MISR cameras, and also indicate which cameras are excluded due to potential sunglint contamination. Consistent with the results reported in Kalashnikova and Kahn (2006) who performed sensitivity studies for a single, mid-latitude viewing geometry - the different panels in Fig. 11 show that the MISR cameras typically sample scattering angles greater than $130^{\circ}$, where the phase functions for Particle 19 and the size-equivalent sphere are significantly different. This, in turn, implies that it is unlikely that the size-equivalent sphere will be confused with the non-spherical dust particle in the MISR Dark Water retrieval. The exception is the viewing geometry for Saharan dust transport shown in Fig. 11a, where the cameras that sample the largest scattering angles are excluded due to potential sunglint contamination. By construction, the phase function of the backscatter-equivalent sphere, however, looks very similar to the phase function of Particle 19 in all four different viewing geometries, and it is highly unlikely that this represents a frequently occurring natural particle.
Besides spherical particles, other non-spherical particles not contained in the existing MISR SMART could potentially be confused with the non-spherical dust models by the MISR Dark Water retrieval algorithm. In this case, the hypothesis is that the "true" aerosols are non-spherical, but not dust, yet the MISR dust model again serves as the closest analogue. Likely candidates are cirrus particles, which were examined in a sensitivity study by Pierce et al. (2010), who found that under various conditions, cirrus particles could not necessarily be distinguished from the MISR non-spherical dust models. The cirrus models used in that study were developed based on the work of Baum et al. (2005a,b), and text files containing the phase functions were obtained from http:// www.ssec.wisc.edu/ baum/Cirrus/MISR_Models.html. The models consist of different ice crystal habits that depend on the size range used in the integration over the size distribution and include droxtals, 3-D bullet rosettes, solid columns, plates, hollow columns, and aggregates. Following Pierce et al. (2010), three different cirrus models were selected, corresponding to three different effective diameters $\left(D_{\mathrm{e}}\right): 10,40$, and $100 \mu \mathrm{m}$.

Figure 12 plots the phase functions for the two MISR nonspherical dust models and the three cirrus models. The cirrus models are distinguished by halo peaks in the phase function at scattering angles of 22 and $46^{\circ}$, scattering angles too 

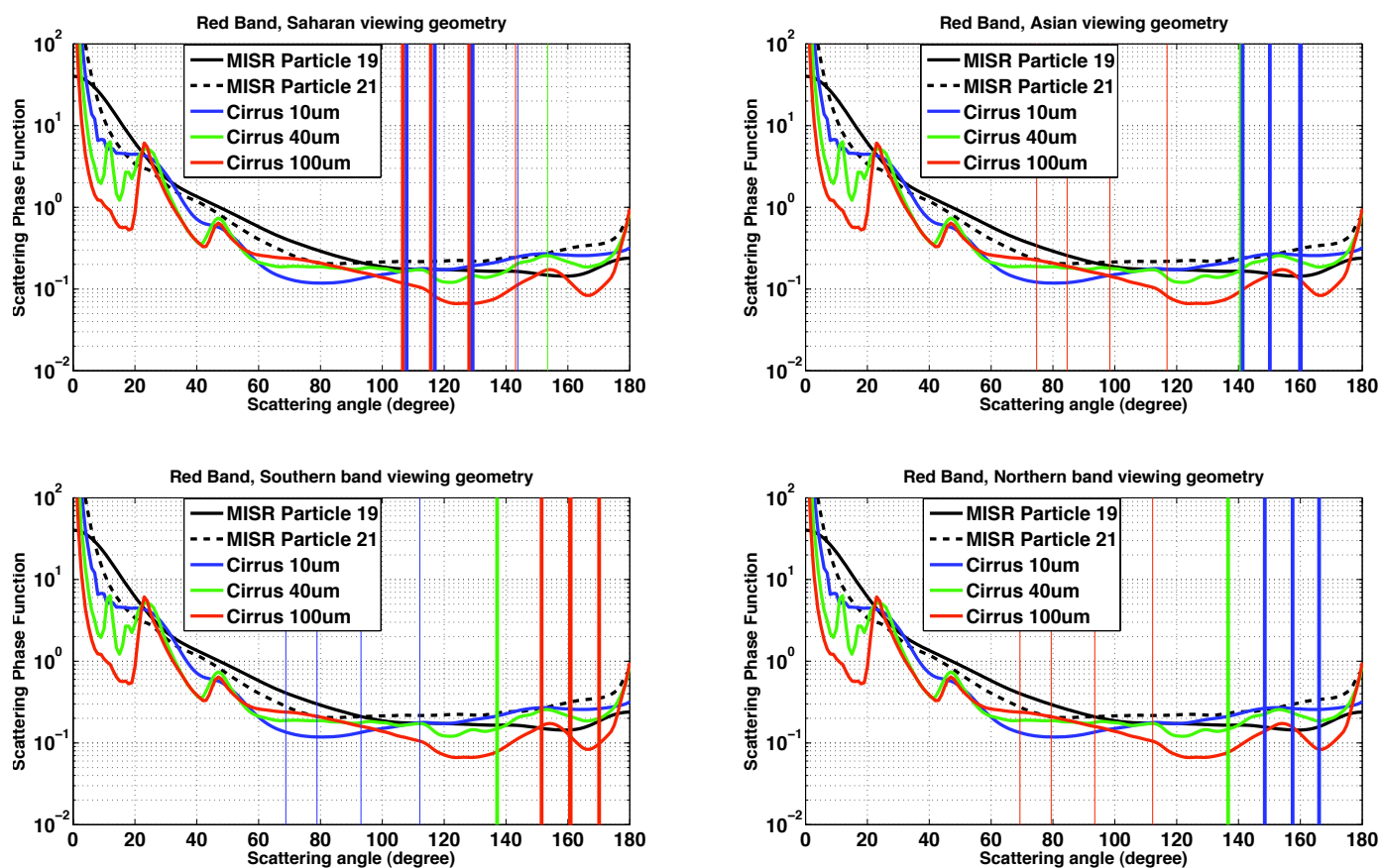

Fig. 12. Same as Fig. 11 but comparing MISR Particle 19 and Particle 21 (coarse dust) with three cirrus particles: $D_{\mathrm{e}}=10 \mu \mathrm{m}, D_{\mathrm{e}}=40 \mu \mathrm{m}$, and $D_{\mathrm{e}}=100 \mu \mathrm{m}$.

forward to be observed by any MISR camera. Unlike the case for the spherical particles, the complicated structure of the cirrus scattering phase functions compared with the phase functions of the dust models, combined with the MISR sampling in the different geometries, makes it difficult to immediately tell from these plots alone whether or not the cirrus particles are distinguishable from the dust particles. It is clear, for instance, that the phase functions for the cirrus particles with $D_{\mathrm{e}}=40 \mu \mathrm{m}$ and $100 \mu \mathrm{m}$ overlap the phase function for MISR Particle 19 at a number of different scattering angles, some of which happen to be precisely those sampled in the enhanced non-sphericity bands in both the Northern and Southern Hemisphere, as shown in Fig. 12c and 12d.

The results of this section show that, while it is unlikely that a size-equivalent spherical particle is being retrieved as a non-spherical dust particle, it is still possible that the backscatter-equivalent sphere and/or cirrus particles are potentially confused with the existing MISR non-spherical dust models by the V22 Dark Water retrieval algorithm, resulting in the unexpected enhanced bands of particle non-sphericity seen in Fig. 8. However, examination of the phase functions alone is insufficient to completely answer this question. A more complete analysis that treats the details of how the MISR V22 Dark Water retrieval algorithm actually works is required, and is introduced in the next section.

\section{Operational MISR Dark Water algorithm particle selection}

The basic approach of the V22 MISR Dark Water retrieval is to assemble a set of aerosol models considered to be representative of the aerosol types likely to be found over the globe into a LUT, which is the SMART. The algorithm determines which models and corresponding AODs satisfy a particular set of four "goodness-of-fit" $\left(\chi^{2}\right)$ criteria by comparing the MISR observations with the results of precalculated, multiple-scattering, radiative transfer simulations stored in the SMART (Martonchik et al., 1998; Kahn et al., 2001; Diner et al., 2002; Martonchik et al., 2002, 2009). As described previously, each aerosol model ("particle") in the MISR SMART is defined by a number-weighted, lognormal distribution that defines the particle size, a particle shape, a spectral complex index of refraction, and a vertical distribution in the atmosphere. Up to three aerosol particles can comprise an aerosol "mixture," with the fractional optical depths of the mixture components prespecified. In this section, we introduce the $\chi^{2}$ tests for goodness of fit and show how these are used by the MISR V22 Dark Water retrieval algorithm to determine the AOD and discriminate aerosol types. Additional discussion of the operational MISR Dark Water aerosol retrieval algorithm can be found in Martonchik et al. (1998), Martonchik et al. (2002), Diner et al. (2005), and Kahn et al. (2007a), but note that some details in these references are for earlier implementations of the algorithm, and are therefore out of date. This section contains 
a description of the important aspects of the current operational V22 algorithm.

\subsection{MISR V22 Dark Water algorithm distinguishability criteria}

The MISR instrument measures top-of-atmosphere (TOA) radiances, $I$, with units of $\mathrm{Wm}^{-2} \mathrm{sr}^{-1} \mu \mathrm{m}^{-1}$. These radiances are normalized by dividing by the extraterrestrial solar irradiance for each wavelength, $F_{0}$, and multiplying by $\pi$ to yield TOA equivalent reflectances, $\rho=\pi I / F_{0}$, which effectively compare the observed radiances to the radiance of an ideal Lambertian reflector illuminated at normal incidence. Note that equivalent reflectances are related to bidirectional reflectance factors, BRFs, through the relation $\rho=$ $\mu_{0} \times \mathrm{BRF}$, where $\mu_{0}$ is the cosine of the solar zenith angle (Kahn et al., 2007a).

The V22 Dark Water retrieval algorithm compares the TOA equivalent reflectances observed by MISR from all valid cameras and wavelengths to a set of precalculated equivalent reflectances for each aerosol model contained in the current MISR SMART. The construction of the MISR SMART is described in Martonchik et al. (1998). Full multiple-scattering radiative transfer calculations are performed based on the scalar matrix operator method (Grant and Hunt, 1968). Rayleigh scattering, including a correction for polarization, is included, and aerosols are described by a base, top, scale height, and AOD. Ozone and out-of-band corrections are done as a preprocessing step (Martonchik et al., 1998; Kahn et al., 2007a).

The AOD for each aerosol model is found by minimizing a $\chi^{2}$ goodness-of-fit parameter (e.g., Bevington and Robinson, 2003). The particular metric used at this stage in the process is known as $\chi_{\text {abs }}^{2}$ because it depends on the absolute reflectance (Kahn et al., 1997, 2001). $\chi_{\mathrm{abs}}^{2}$, which depends on the model AOD, $\tau$, is determined from the equation

$$
\chi_{\mathrm{abs}}^{2}(\tau)=\frac{\sum_{l=1}^{4} w_{l} \cdot\left\{\sum_{j=1}^{9} w_{j} \cdot v(l, j) \cdot \frac{\left[\rho_{\mathrm{MISR}}(l, j)-\rho_{\mathrm{model}}(i, j)\right]^{2}}{\sigma_{\mathrm{abs}}^{2}(l, j)}\right\}}{\sum_{l=1}^{4} w_{l} \cdot\left[\sum_{j=1}^{9} w_{j} \cdot v(l, j)\right]}
$$

where $\rho_{\text {MISR }}$ represents the equivalent reflectance at wavelength $l$ and camera $j$ from the darkest $1.1 \mathrm{~km}$ subregion in the $17.6 \mathrm{~km} \times 17.6 \mathrm{~km}$ region used for the retrieval, $\rho_{\text {model }}$ are the TOA equivalent reflectances for the candidate aerosol model, and $\sigma_{\mathrm{abs}}$ accounts for the absolute radiometric uncertainty assumed for the MISR instrument. Valid observations for a particular wavelength and camera are given a weight $v(i, j)=1$. The weighting parameters, $w_{j}$, which could in principle vary by camera, are presently given a value of 1 for all cameras. The information is also weighted by wavelength, $w_{l}$. For AODs less than 0.5 , only the red and NIR wavelengths, where the water surface is assumed to have negligible water-leaving radiance, are used in the V22 Dark Water retrieval (Martonchik et al., 1998). In Eq. (2) the weights for these wavelengths are always set to 1 . The green wavelength is introduced when the AOD exceeds 0.5, and the weight, $w_{2}$, increases linearly up to an AOD of 1.00 when $w_{2}=1$. The blue wavelength is similarly introduced when the AOD exceeds 0.75 , with full weight, $w_{1}=1$, when the $\mathrm{AOD}=1.50$. In the V22 algorithm, if the minimum $\chi_{\mathrm{abs}}^{2}$, determined through a parabolic fitting procedure (Martonchik et al., 1998), is less than 2, the model is considered "passing" at this stage in the process and the model and associated AOD are retained for further analysis.

A detailed discussion of the characteristics of the $\chi_{\mathrm{abs}}^{2}$ metric and the dependence on the choice of the threshold can be found in Kahn et al. (2001). However, it is worth noting here the relationship between $\chi_{\mathrm{abs}}^{2}$ and the "standard score" or " $z$ score" described in Kalashnikova et al. (2011). The $z$ score is simply the absolute value of the difference between a single measurement and a model calculation, which is then divided by the assumed noise in the measurement represented as a standard deviation. In other words,

$z=\frac{\left|\rho_{\text {MISR }}-\rho_{\text {model }}\right|}{\sigma}$.

Comparison of Eqs. (2) and (3) shows that the term in braces in Eq. (2) is simply the square of the $z$ score summed over angles. The $z$ score has a simple statistical interpretation as the difference between a sample, $\rho_{\text {model }}$, and the mean of a population, $\rho_{\text {MISR }}$, in units of the standard deviation of the population, $\sigma$ (Kalashnikova et al., 2011). If the errors are normally distributed, when $z=1$ the probability is $31.7 \%$ that the sample does not belong to the parent population. If $z=2$, the probability that the observation is consistent with the model is only $4.6 \%$. This statistically derived conclusion depends in a fundamental way on the value of the denominator in Eq. (3), which should represent the expected noise in the observations based on an analysis of instrument performance. This choice will be discussed further in the following section.

The MISR V22 Dark Water retrieval algorithm employs three additional $\chi^{2}$ metrics that are designed to take greater advantage of MISR's unique observational approach to improve aerosol particle discrimination (Kahn et al., 1997; Martonchik et al., 1998; Kahn et al., 2001).

The first $\chi^{2}$ metric is designed to emphasize spectral differences between two aerosol models. In the MISR V22 Dark Water retrieval algorithm this metric is defined as

$$
\chi_{\text {spec }}^{2}(\tau)=\frac{\sum_{l=1, l \neq 3}^{4} w_{l} \cdot \sum_{j=1}^{9} v_{j}(l, \operatorname{Red}) \cdot \frac{\left[\frac{\rho_{\text {MISRR }}(l, j)}{\rho_{\text {MISR Red }}(j)}-\frac{\rho_{\text {moded }}(l, j)}{\rho_{\text {model, Red }}(j)}\right]^{2}}{\sigma_{\text {spec }}(l, j)}}{\sum_{l=1, l \neq 3}^{4} w_{l} \cdot \sum_{j=1}^{9} v_{j}(l, \text { Red })},
$$

where the summation is over the nine MISR cameras, $w$ is set to unity for all cameras, and $v_{j}(l$, Red) has a value of 1 if both the red wavelength and the comparison wavelength 
have valid data for that camera. The uncertainty, which is a function of the camera, $j$, is expressed as

$\sigma_{\text {spec }}(l, j)=0.05 \cdot \frac{\rho_{\text {MISR }}(l, j)}{\rho_{\text {MISR }, \operatorname{Red}}(j)}$.

In the MISR V22 Dark Water aerosol retrieval algorithm, $\chi_{\text {spec }}^{2}$ compares the spectral behavior of the observations at the red wavelength to the observations at the other wavelengths, taking advantage of the relatively better wavelengthto-wavelength calibration of the MISR instrument compared to the absolute calibration (Martonchik et al., 1998; Kahn et al., 2001). If the value of $\chi_{\mathrm{spec}}^{2}$ is greater than 3 , the model is no longer considered to be an acceptable match to the MISR observations, regardless of the value of $\chi_{\mathrm{abs}}^{2}$.

The next $\chi^{2}$ metric is intended to take advantage of MISR's multiangle observation strategy, and emphasizes angular differences in the way different aerosol models scatter light (Kahn et al., 1997; Martonchik et al., 1998; Kahn et al., 2001). In the MISR V22 Dark Water aerosol retrieval this metric is defined as

$\chi_{\text {geom }}^{2}(\tau)=\frac{\sum_{l=3}^{4} w_{l} \cdot\left\{\sum_{j=1}^{9} w_{j} \cdot v(l, j) \cdot \frac{\left[\frac{\rho_{\text {MISR }}(l, j)}{\rho_{\text {MISR }}(l, a \text { allcam })}-\frac{\rho_{\text {model }}(l, j)}{\rho_{\text {model }}(\text {,allcam })}\right]^{2}}{\sigma_{\text {geom }}^{2}(l, j)}\right\}}{\sum_{l=3}^{4} w_{l} \cdot\left[\sum_{j=1}^{9} w_{j} \cdot v(l, j)\right]}$,

where $w_{l}$ is set to unity for all wavelengths, $l ; w_{j}$ is set to unity for all cameras, $j$; and $v$ has a weight of 1 if the combination of wavelength and camera is simultaneously valid. The equivalent reflectances are normalized to the mean value over all cameras within each wavelength using the following expression:

$\rho_{\mathrm{MISR}}(l$, allcam $)=\frac{\sum_{j=1}^{9} w_{j} \cdot v(l, j) \cdot \rho_{\mathrm{MISR}}(l, j)}{\sum_{j=1}^{9} w_{j} \cdot v(l, j)}$.

A similar expression is used to determine $\rho_{\text {model }}$. The uncertainty in Eq. (6) is given by the relation

$\sigma_{\text {geom }}(l, j)=0.05 \cdot \frac{\rho_{\text {MISR }}(l, j)}{\rho_{\text {MISR }}(l, \text { allcam })}$.

$\chi_{\text {geom }}^{2}$ is very sensitive to particle shape, as noted by Kahn et al. (1997) based on initial sensitivity studies of MISR's ability to distinguish spherical and non-spherical aerosols, and, like $\chi_{\text {spec }}^{2}$, this metric leverages better relative camerato-camera calibration compared to the absolute calibration of the instrument. In the MISR V22 Dark Water aerosol retrieval algorithm, a value of $\chi_{\text {geom }}^{2}$ greater than 3 indicates that the model is not an acceptable fit to the MISR observations.

The final $\chi^{2}$ metric is the maximum deviation parameter. $\chi_{\text {maxdev }}^{2}$, which is simply the maximum value attained for any wavelength or camera in the calculation of $\chi_{\mathrm{abs}}^{2}$ : $\chi_{\operatorname{maxdev}}^{2}(\tau)=\max \left\{\frac{\left[\rho_{\mathrm{MISR}}(l, j)-\rho_{\text {model }}(i, j)\right]^{2}}{\sigma_{\text {abs }}^{2}(l, j)}\right\}$.

To be considered valid, a candidate aerosol cannot have a value of $\chi_{\text {maxdev }}^{2}$ greater than 5 in the MISR V22 Dark Water aerosol retrieval algorithm. The large magnitude of this threshold limits the sensitivity of this particular metric.

Those models and associated AODs, determined through the calculation of $\chi_{\mathrm{abs}}^{2}$, that meet all four of the threshold criteria are considered "passing" aerosol mixtures. The average of the AODs of these passing mixtures is reported as the RegBestEstimateSpectralOptDepth field in the MISR V22 aerosol product. The model with the lowest value of each $\chi^{2}$ metric, determined through a weighting procedure, and its associated AOD are reported as the RegLowestResidMixture and RegLowestResidSpectralOptDepth, respectively (Martonchik et al., 1998; Martonchik et al., 2002; Diner et al., 2005; Kahn et al., 2007a).

\subsection{Effect of the radiometric "floor" on calculation of $\chi_{\text {abs }}^{2}$}

In the definition of $\chi_{\mathrm{abs}}^{2}$ given in Eq. (2), a term, $\sigma_{\mathrm{abs}}$, appears that expresses the uncertainty in the absolute radiance measurements made by the instrument. The absolute calibration of MISR, both pre- and postlaunch, has been evaluated in a number of publications (Bruegge et al., 1998a,b; Kahn et al., 2005b; Bruegge et al., 2007; Lyapustin et al., 2007; Lallart et al., 2008). These studies generally conclude that the absolute calibration uncertainty for any camera is around $3 \%$, with the camera-to-camera agreement being substantially better, about $1 \%$. Adopting a conservative estimate of $5 \%$ for the calibration uncertainty, the value for $\sigma_{\mathrm{abs}}$ in Eq. (2) used by the V22 Dark Water algorithm is determined from the equation

$\sigma_{\text {abs }}(l, j)=0.05 \cdot \max \left[\rho_{\operatorname{MISR}}(l, j), 0.04\right]$.

The leading term on the right-hand side of Eq. (10) represents the assumed $5 \%$ radiometric calibration uncertainty. The term in brackets indicates that the procedure is to take the maximum of either the MISR-observed TOA equivalent reflectance or 0.04 , which acts as an absolute minimum (i.e., a radiometric "floor").

This floor was included in the algorithm under the conservative assumption that measurement or environmental noise could limit the minimum value of $\sigma_{\mathrm{abs}}$. However, experience shows the noise levels in the MISR data to be very low. The presence of the floor is also problematic on theoretical grounds. For example, when the aerosol optical depth of the atmosphere is small, radiative transfer can be performed assuming single scattering dominates. The standard equation for single scattering in a one-dimensional, plane-parallel atmosphere can be written as

$$
\frac{\pi I}{\mu_{0} F_{0}}=\frac{\varpi_{0}}{4\left(\mu+\mu_{0}\right)} P(\Theta)\left\{1-\exp \left[-\tau\left(\frac{1}{\mu}+\frac{1}{\mu_{0}}\right)\right]\right\} \text {, }
$$


(a) MISR dark water algorithm

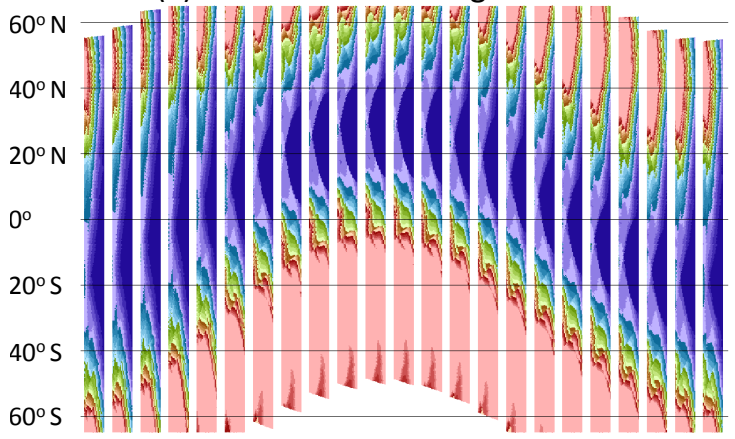

Jan Feb Mar Apr May Jun Jul Aug Sept Oct Nov Dec (b) no min radiance criteria in $\chi_{\text {abs }}$

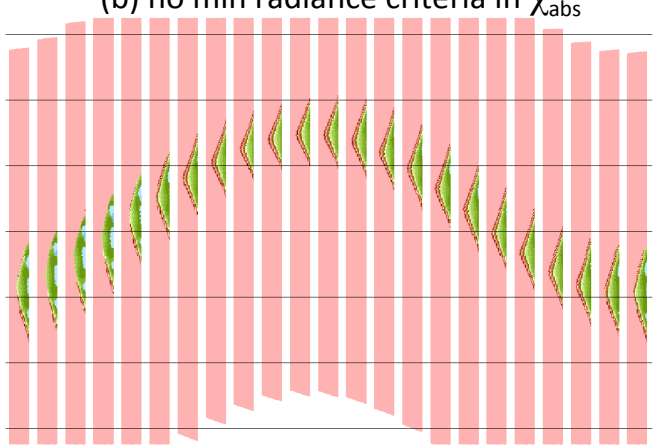

Jan Feb Mar Apr May Jun Jul Aug Sept Oct Nov Dec

Single scattering

approximation

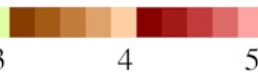

$\mathrm{AOD}=0.05$

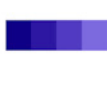

123

$\chi_{\text {abs }}$ Particle 19 - Size-equivalent sphere

Fig. 13. $\chi_{\mathrm{abs}}^{2}$ calculated for MISR red and NIR wavelengths only, comparing MISR Particle 19 and the size-equivalent spherical particle, presented as a function of latitude and time in the same manner as Fig. 6. The plots take into account the changing viewing geometry, using the single-scattering approximation for $\mathrm{AOD}=0.05$ assuming a black surface, including the effect of cameras excluded due to potential sunglint contamination. In the MISR V22 Dark Water retrieval algorithm, $\chi_{\mathrm{abs}}^{2}$ values less than 2 mean that the particles are indistinguishable from one another. Larger values of $\chi_{\mathrm{abs}}^{2}$ indicate greater distinguishability. (a) Calculation of $\chi_{\mathrm{abs}}^{2}$ as done in the V22 Dark Water algorithm. (b) Calculation of $\chi_{\mathrm{abs}}^{2}$ excluding the radiometric floor.

where $I$ is the radiance, $\mu_{0}$ is the cosine of the solar zenith angle, $F_{0}$ is the extraterrestrial solar irradiance, $\varpi_{0}$ is the single-scattering albedo, $\mu$ is the cosine of the camera viewing zenith angle, $P(\Theta)$ is the phase function that depends on the scattering angle $\Theta$, and $\tau$ is the total column AOD (Liou, 2002). The left-hand side of Eq. (11) is simply the BRF at the TOA. The utility of the single-scattering approximation is that it can be used to analytically calculate TOA equivalent reflectances for each MISR camera given the viewing geometry, AOD, and the phase function of a particle.

Solving this equation for the AOD leads to the following expression:

$$
\tau=-\frac{\ln \left[1-\frac{4\left(\mu+\mu_{0}\right) \rho}{\mu_{0} \varpi_{0} P(\Theta)}\right]}{\left(\frac{1}{\mu}+\frac{1}{\mu_{0}}\right)} .
$$

For this expression to be mathematically valid, the term in square brackets on the right-hand side must be greater than zero. This, in turn, leads to the relation

$$
\frac{\mu_{0} \varpi_{0} P(\Theta)}{4\left(\mu+\mu_{0}\right)}>\rho,
$$

which provides an upper bound to the TOA equivalent reflectance, assuming the validity of the single-scattering approximation. Taking the red wavelength phase function for MISR Particle 19 (medium dust) as an example, this relation shows that for a nadir-viewing camera $(\mu=1)$, the maximum value the TOA equivalent reflectance can reach for any solar zenith angle is less than 0.03 , which is smaller than the value of 0.04 appearing in Eq. (10). This means that the radiometric floor is likely to play a substantial, but not necessarily beneficial, role in the sensitivity of the MISR V22 Dark Water retrieval, particularly for low AOD, when the single-scattering approximation is most applicable.

The consequences of the presence of the radiometric floor on the calculation of $\chi_{\mathrm{abs}}^{2}$ and the sensitivity of the MISR Dark Water retrieval are explored further in Fig. 13. The two panels compare the calculation of $\chi_{\mathrm{abs}}^{2}$ with and without including the radiometric floor in determining $\sigma_{\text {abss }}$. As was done in Fig. 6, each individual strip represents the full cross-swath MISR viewing geometry. This geometry was used, along with the single-scattering approximation, to calculate TOA equivalent reflectances for the MISR red and NIR wavelengths for all valid MISR cameras for two different particles, assuming an AOD of 0.05 and a black surface. The particles used for the results shown here are MISR Particle 19 (medium dust) and the size-equivalent sphere. Values for $\chi_{\mathrm{abs}}^{2}$ were determined using Eq. (2) for each location assuming the "true" atmosphere contained only Particle 19. Small values of $\chi_{\mathrm{abs}}^{2}$ (shown in purple and blue colors) imply that the particles are indistinguishable from one another based on this metric alone. Recall that the threshold adopted in the MISR V22 Dark Water algorithm for an acceptable match is $\chi_{\mathrm{abs}}^{2}<2$. Green and red colors indicate greater distinguishability.

Figure 13a uses the single-scattering approximation but calculates $\chi_{\text {abs }}^{2}$ including the radiometric floor in the same way as it is done in the V22 Dark Water retrieval algorithm. Surprisingly, given Fig. 11 and the associated discussion, this figure implies there is a significant portion 
of the MISR seasonally variable viewing geometry within which Particle 19 cannot be distinguished from the sizeequivalent sphere. According to Eq. (11), the TOA equivalent reflectances depend directly on the particle phase function under the assumption of single scattering. Therefore, it is curious that the large differences between the non-spherical and spherical phase functions shown in Fig. 11 do not translate directly into sensitivity in terms of $\chi_{\mathrm{abs}}^{2}$. Figure $13 \mathrm{~b}$ reveals that the radiometric floor is the reason for this lack of sensitivity. Once the radiometric floor has been eliminated from the determination of $\sigma_{\mathrm{abs}}$, the non-spherical dust and sizeequivalent sphere are seen to have $\chi_{\mathrm{abs}}^{2}$ significantly larger than 5 for most MISR viewing geometries. The exception is a small region near the subsolar point where the $\chi_{\mathrm{abs}}^{2}$ values are as small as 3, which is consistent with the discussion related to Fig. 11.

A number of previous publications (Kahn et al., 1998, 2001, 2005a, 2010; Kalashnikova and Kahn, 2006) have noted decreased sensitivity to particle microphysics (i.e., particle shape and size) for MISR aerosol retrievals when the AOD is less than about 0.15 or 0.2 . The argument is made that this reduced sensitivity is expected due to "an overall lack of information about particle properties when the atmosphere contains few aerosols" (Kalashnikova and Kahn, 2006) or "owing to increased relative contributions from surface reflectance uncertainties, unmasked cloud, etc." (Kahn et al., 2010). While these assertions may be correct in certain circumstances, Fig. 13 suggests another possibility. Careful examination of the observed MISR TOA equivalent reflectances for a number of situations when the scene is very dark and cloud-free reveals the radiometric noise to be extremely small, even when $\rho_{\text {MISR }}<0.04$. What Fig. 13a shows then is that the radiometric floor artificially reduces the sensitivity of $\chi_{\mathrm{abs}}^{2}$ by enforcing a value for $\sigma_{\mathrm{abs}}$ that is larger than necessary. This is confirmed in Fig. 13b, in which sensitivity to particle shape is recovered for nearly every available MISR viewing geometry. Therefore, the argument can be made that when the total AOD is low, and the atmosphere is closest to single scattering, the MISR Dark Water retrieval should have the greatest sensitivity to differences in the scattering phase functions - differences that are reduced when significant multiple scattering occurs. This suggests that the MISR V22 Dark Water aerosol retrieval algorithm does not presently take full advantage of the sensitivity of the MISR instrument for retrieving particle microphysics for small AOD. Further work is required, however, to fully demonstrate this capability for operational aerosol retrievals.

\section{Sensitivity of operational MISR Dark Water algorithm to aerosol particle shape}

Section 4 discussed MISR sensitivity to different particles based simply on an examination of their scattering phase functions and the specific scattering angles observed by the
MISR cameras for different geometries. This analysis alone was insufficient to answer the question of whether or not aerosol particles missing from the current MISR SMART dataset are the reason for the non-spherical artifacts observed in the global MISR V22 non-spherical AOD fraction climatology. In this section we consider the performance of the Dark Water retrieval algorithm as it is implemented operationally, the influence of the changing MISR viewing geometry over the orbit and throughout the year, and the impact of excluding different cameras due to potential contamination by sunglint. This discussion extends the work of Kahn et al. (1997), Kalashnikova et al. (2005), Kalashnikova and Kahn (2006), and Pierce et al. (2010), which were primarily sensitivity studies carried out over a limited but representative range of conditions.

\subsection{Single-scattering approximation and $\chi_{\mathrm{abs}}^{2}$}

In this section we utilize the single-scattering approximation as a method for analyzing the behavior of the $\chi_{\mathrm{abs}}^{2}$ metric when comparing different particles. Although the singlescattering approximation is not strictly correct, it is useful because it is a procedure for analytically calculating TOA equivalent reflectances for a plane-parallel atmosphere without resorting to a full multiple-scattering radiative transfer code. This provides a quick way of determining which particles are likely to be distinguishable from one another and which are likely to be indistinguishable given the actual MISR viewing geometry. The effect of including full multiple scattering, as is done in the MISR V22 Dark Water aerosol retrieval, will be discussed in the next section.

Throughout the remaining discussions, we retain the radiometric floor in calculations of $\sigma_{\text {abs }}$ so as to evaluate the sensitivity of $\chi_{\mathrm{abs}}^{2}$ as it is implemented operationally in version 22. Figures 14 and 15 show calculations of $\chi_{\mathrm{abs}}^{2}$ presented in the same manner as Fig. 13, using the singlescattering approximation but comparing MISR Particle 19 against the spherical and non-spherical models whose phase functions are shown in Figs. 11 and 12. In all cases, an AOD of 0.05 and a black surface are assumed for the particles. Each panel in these figures can be compared with Fig. 8 to determine whether or not indistinguishability in terms of the $\chi_{\mathrm{abs}}^{2}$ metric between a particle not in the MISR SMART and MISR Particle 19 (medium dust) could help explain the observed bands of enhanced particle non-sphericity. Recall that purple and blue colors correspond to locations with $\chi_{\mathrm{abs}}^{2}$ values low enough to be considered acceptable matches by the MISR V22 Dark Water retrieval algorithm. Figure 14a and c show bands with poor distinguishability, but these occur around the subsolar geometry and are not consistent with the non-spherical bands in Fig. 8. The backscatter-equivalent sphere, on the other hand, has very low values of $\chi_{\mathrm{abs}}^{2}$ for nearly every viewing geometry, and therefore remains a potential candidate for causing the observed bands of enhanced non-sphericity. In other words, if a naturally occurring, 
(a) $\chi_{\text {abs }}$ Size-equivalent sphere - Particle19

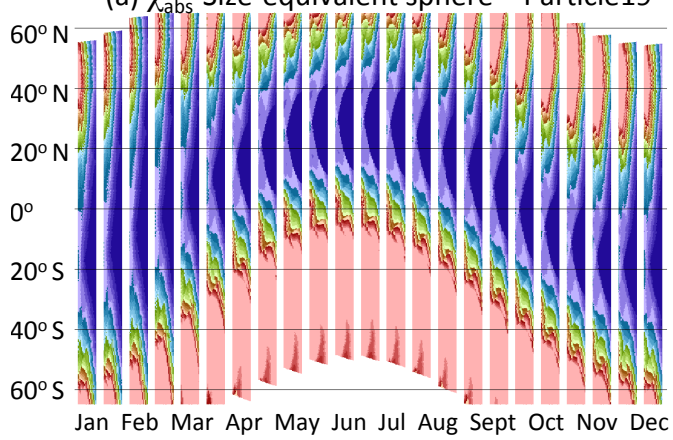

(c) $\chi_{\text {abs }}$ Particle 21 - Particle19

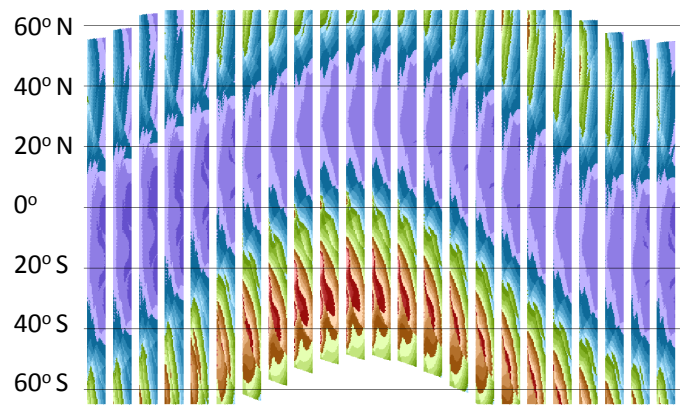

Jan Feb Mar Apr May Jun Jul Aug Sept Oct Nov Dec (b) $\chi_{\text {abs }}$ Backscatter-equivalent sphere - Particle19

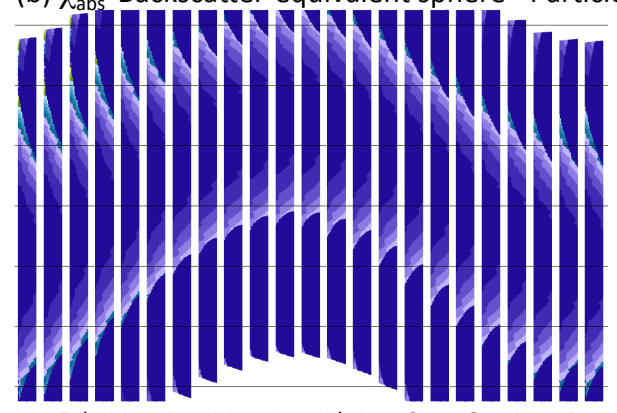

Jan Feb Mar Apr May Jun Jul Aug Sept Oct Nov Dec

Single scattering approximation

$A O D=0.05$

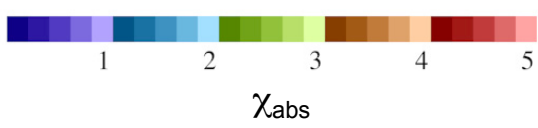

Fig. 14. $\chi_{\mathrm{abs}}^{2}$ calculated for MISR red and NIR wavelengths only, as in Fig. 13, comparing MISR Particle 19 and the three other particles using the single-scattering approximation: (a) Size-equivalent sphere (identical to Fig. 13a), (b) backscatter-equivalent sphere, and (c) MISR Particle 21.

spherical aerosol similar to the backscatter-equivalent sphere happened to be present over the ocean, it is possible that the MISR V22 Dark Water algorithm would retrieve a nonspherical aerosol (Particle 19) at these locations instead. This is because the non-spherical particle is the only one in the existing MISR SMART that provides an adequate fit to the observations.

Figure 15 shows that in the case of the cirrus models, the particles with 10 and $40 \mu \mathrm{m}$ effective diameters are less distinguishable from Particle 19 around the subsolar point, which is not where the bands of enhanced non-sphericity appear in Fig. 8. However, the $100 \mu \mathrm{m}$ effective diameter particle shows the opposite behavior, with greater distinguishability from Particle 19 around the subsolar point, but reduced distinguishability at higher latitudes in precisely those locations where the bands of enhanced non-sphericity appear in Fig. 8. This suggests that larger cirrus particles may provide another explanation for the appearance of the bands of enhanced non-sphericity, while the smaller cirrus particles are less likely to be candidates.

Based on this analysis, we have found that it is possible that aerosol optical models missing from the current MISR SMART are the underlying cause for the bands of enhanced non-sphericity appearing in seasonal plots of MISR V22 non-spherical AOD fraction. The analysis also shows that missing aerosol models, such as the backscatter-equivalent sphere, if present in the atmosphere, could be retrieved as non-spherical aerosols at low optical depths - but this occurs everywhere, not only in specific bands. We have further narrowed the candidate models down to a backscatteringequivalent sphere and a cirrus model with an effective diameter around $100 \mu \mathrm{m}$. In the next section we investigate these two models further using full multiple-scattering radiative transfer calculations as is done by the MISR V22 Dark Water retrieval algorithm.

\subsection{Full multiple-scattering radiative transfer and additional $\chi^{2}$ metrics}

To more completely evaluate the potential of spherical and non-spherical aerosols missing from the current MISR SMART to explain the presence of temporally varying bands of enhanced particle non-sphericity observed in the MISR V22 aerosol climatology over the global oceans, we perform full multiple-scattering calculations for atmospheres containing MISR Particle 19 (medium dust), backscatter-equivalent spherical particles, and cirrus particles with $D_{\mathrm{e}}=100 \mu \mathrm{m}$.

Because generation of a completely new SMART is a complex and computationally involved task, previous sensitivity studies for non-spherical particles such as Kalashnikova and Kahn (2006) and Pierce et al. (2010) have employed the MISR Research Aerosol Retrieval approach described in 
(a) $\chi_{\text {abs }}$ Cirrus $10 u m$ - Particle19

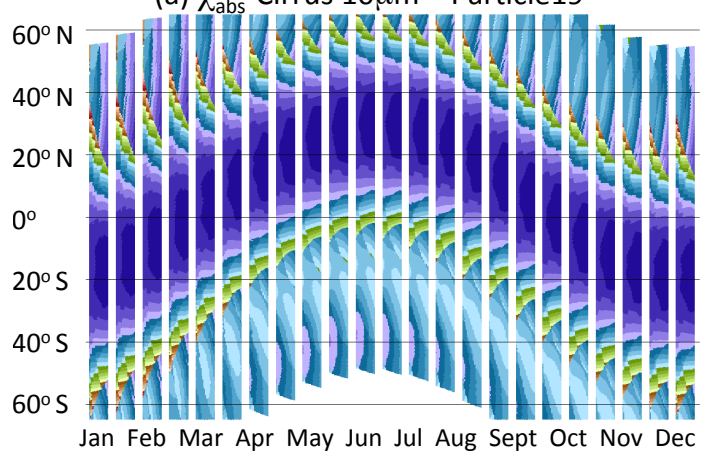

(c) $\chi_{\text {abs }}$ Cirrus $100 \mu \mathrm{m}$ - Particle19

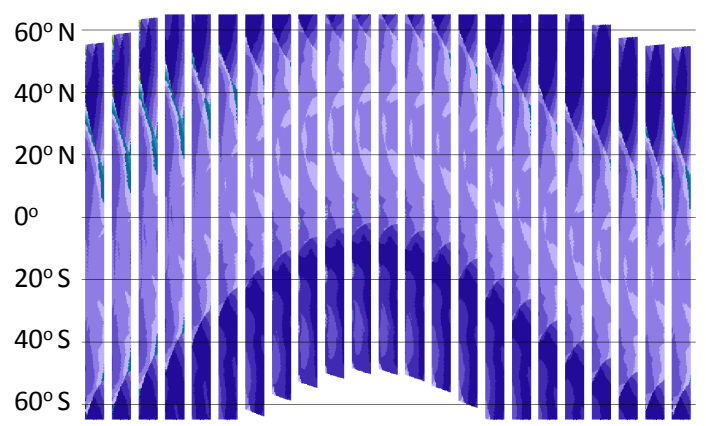

(b) $\chi_{\text {abs }}$ Cirrus $40 \mu \mathrm{m}$ - Particle19

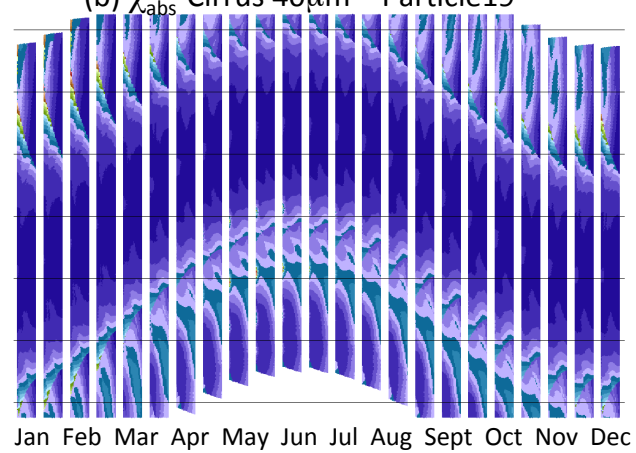

Jan Feb Mar Apr May Jun Jul Aug Sept Oct Nov Dec

Jan Feb Mar Apr May Jun Jul Aug Sept Oct Nov Dec

Single scattering approximation $\mathrm{AOD}=0.05$

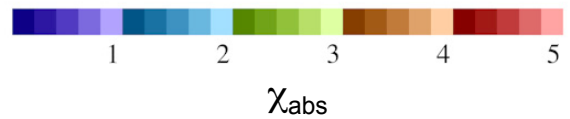

Fig. 15. Same as Fig. 14 but comparing MISR Particle 19 with three cirrus particles: (a) $D_{\mathrm{e}}=10 \mu \mathrm{m}$, (b) $D_{\mathrm{e}}=40 \mu \mathrm{m}$, and (c) $D_{\mathrm{e}}=100 \mu \mathrm{m}$.

Kahn et al. (2001, 2007a). The Research Aerosol Retrieval also uses the scalar matrix operator method, but performs an extensive series of calculations for a wide variety of aerosol particles and mixtures for a single viewing geometry of interest. Here we introduce a third approach that allows for us to explore the complete, seasonally varying MISR observational geometry without the computational cost of generating an entirely new SMART dataset.

For our radiative transfer calculations, we use a vector (polarized) radiative transfer code based on the successiveorder-of-scatter (SOS) method developed for the coupled atmosphere-ocean system (Zhai et al., 2009, 2010). The SOS code provides more flexibility than the existing MISR SMART generation code and can be scripted to calculate results for multiple viewing geometries. The goal of this exercise is to test the role of aerosol models not included in the existing MISR SMART on the appearance of the bands of enhanced particle non-sphericity, rather than fully evaluate how such models would perform once included in the operational MISR Dark Water aerosol retrieval. Therefore, calculations were performed for atmospheres containing only Rayleigh scattering and a single aerosol particle model mixed uniformly within a single layer over a black surface. Rayleigh calculations were done for a molecular scattering atmosphere based on the approach of Bodhaine et al. (1999), which differs somewhat from the Rayleigh optical depth approximation (Russell et al., 1993) used in the generation of the MISR SMART. The Rayleigh optical depths for the two longest MISR wavelengths are 0.04299 (red) and 0.01536 (NIR).

MISR Particle 19 (medium dust) was used as the reference atmosphere and was uniformly mixed with the Rayleigh atmosphere at altitudes between 3 and $6 \mathrm{~km}$. The backscatterequivalent spherical particle was uniformly mixed from 0 to $2 \mathrm{~km}$, and the $100 \mu \mathrm{m}$ effective diameter cirrus particle was uniformly mixed from 10 to $11 \mathrm{~km}$. As noted above, the existing MISR SMART includes a polarization correction for Rayleigh scattering using vector calculations from the code developed by Evans and Stephens (1991). To mimic this behavior, the SOS code was run in vector mode, but the elements of the scattering phase matrix other than the phase function itself, $P_{11}$, were set to zero for the aerosol particles, while the full scattering phase matrix was retained for the Rayleigh scattering portion of the atmosphere. This approach was also necessary because the additional elements of the full scattering phase matrix are not available for MISR Particle 19, for which the phase function alone was originally calculated (Kalashnikova et al., 2005).

In the case of full multiple scattering, the AODs of the different atmospheres become important, as demonstrated in Fig. 16. The left-hand panel shows the calculation of $\chi_{\mathrm{abs}}^{2}$ for an atmosphere containing the backscatter-equivalent sphere 
(a) $A O D=0.10$ (dust) vs. $A O D=0.10$ (sphere)

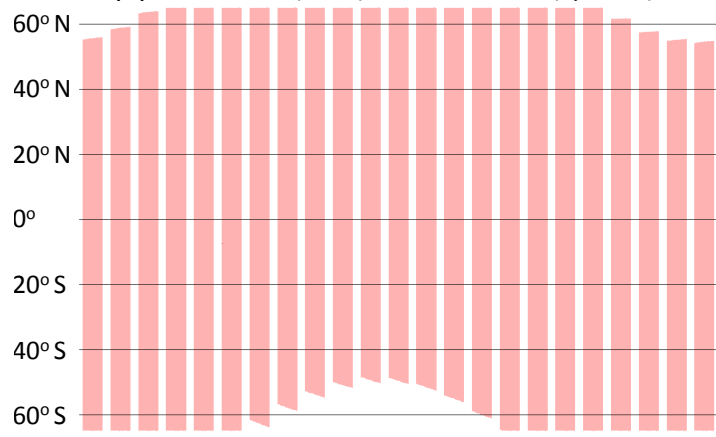

Jan Feb Mar Apr May Jun Jul Aug Sept Oct Nov Dec (b) $A O D=0.10$ (dust) vs. $A O D=0.20$ (sphere)

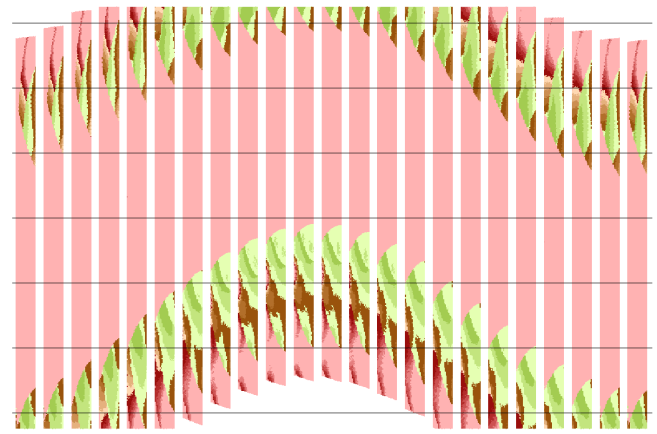

Jan Feb Mar Apr May Jun Jul Aug Sept Oct Nov Dec

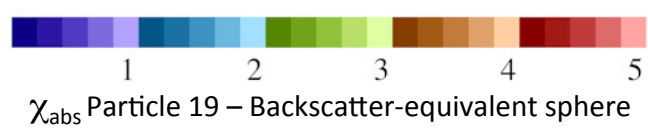

SOS results

$\chi_{\text {abs }}$ Particle 19 - Backscatter-equivalent sphere

Fig. 16. $\chi_{\text {abs }}^{2}$ calculated for MISR red and NIR wavelengths only for a Rayleigh-scattering atmosphere containing only MISR Particle 19 over a black surface and a Rayleigh-scattering atmosphere containing only the backscatter-equivalent spherical particle, as in Fig. 13, but considering full multiple scattering based on SOS radiative transfer calculations. (a) AOD $=0.1$ for both aerosol models, $(\mathbf{b})$ AOD $=0.1$ for Particle 19, and $\mathrm{AOD}=0.2$ for the backscatter-equivalent sphere.

(a) $A O D=0.10$ (dust) vs. $A O D=0.10$ (cirrus)

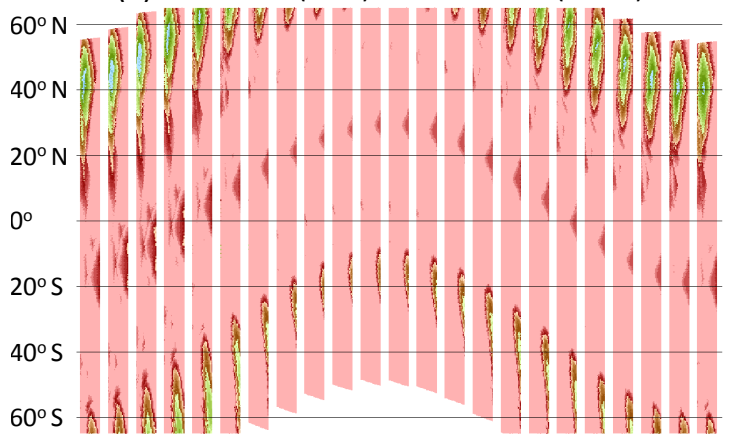

Jan Feb Mar Apr May Jun Jul Aug Sept Oct Nov Dec (b) $A O D=0.10$ (dust) vs. $A O D=0.21$ (cirrus)

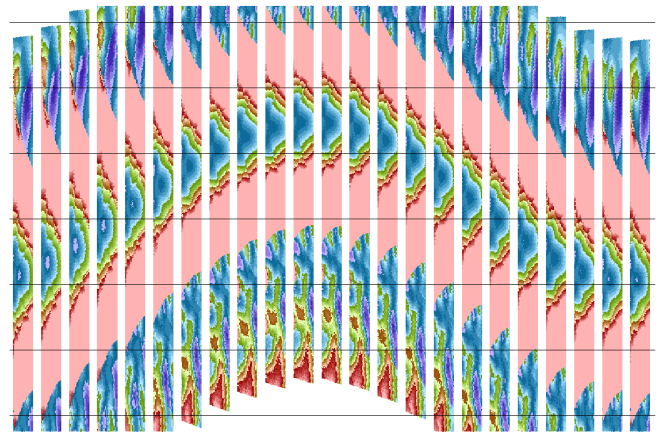

Jan Feb Mar Apr May Jun Jul Aug Sept Oct Nov Dec

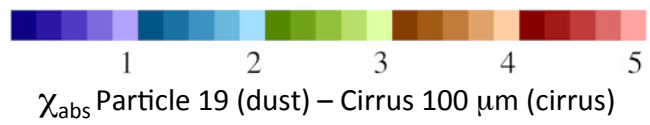

SOS results

Fig. 17. Same as Fig. 16, but comparing an atmosphere with MISR Particle 19 with an atmosphere containing the cirrus model with $D_{\mathrm{e}}=$ $100 \mu \mathrm{m}$. (a) $\mathrm{AOD}=0.1$ for both aerosol models, (b) $\mathrm{AOD}=0.1$ for Particle 19, and $\mathrm{AOD}=0.21$ for the cirrus model.

compared with a reference atmosphere containing MISR Particle 19, both with $\mathrm{AOD}=0.1$. In this case, the atmospheres are distinguishable for every MISR viewing geometry, in contrast to Fig. 14b. By varying the AOD of the test atmosphere containing the backscatter-equivalent sphere in increments of 0.01 , the atmosphere that showed the smallest values of $\chi_{\mathrm{abs}}^{2}$ was found, which is shown in Fig. 16b. In this case, where the AOD of the backscatter-equivalent sphere is 0.2 , bands of reduced sensitivity appear near the latitudes where the bands of enhanced non-sphericity appear in Fig. 8 . Notice that in this case, however, the minimum value of $\chi_{\mathrm{abs}}^{2}$ is greater than 2, indicating that the MISR V22 Dark Water retrieval algorithm would not consider Particle 19 to be an adequate match to the atmosphere containing the backscatter-equivalent sphere. Based on this analysis, it is unlikely that some spherical aerosol particles present in nature, but not included in the MISR aerosol LUT, are being fit by the non-spherical particles in the LUT, thus producing the bands of enhanced non-sphericity.

This leaves the large cirrus model, which is compared with the atmosphere containing MISR Particle 19 in Fig. 17. For the case when the AODs of the two atmospheres are identical, shown in Fig. 17a, bands of reduced $\chi_{\text {abs }}^{2}$ appear, but with values greater than 2 . On the other hand, Fig. 17b shows the result for the case when the cirrus AOD is 0.21 , compared with the reference AOD of 0.1 for the non-spherical dust 

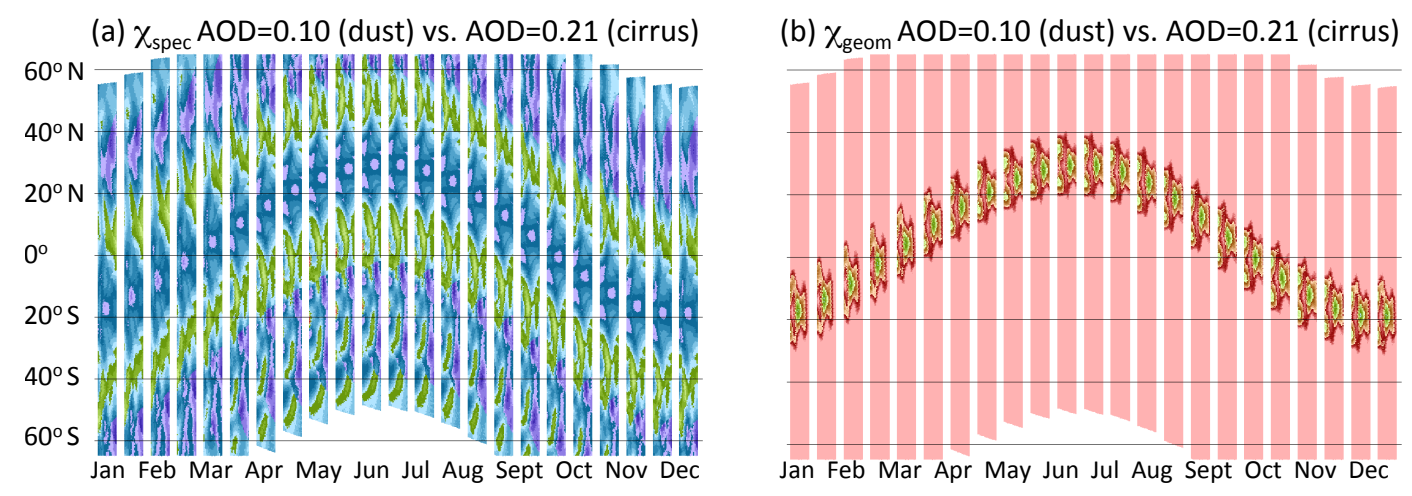

(c) $\chi_{\text {maxdev }} A O D=0.10$ (dust) vs. $A O D=0.21$ (cirrus)

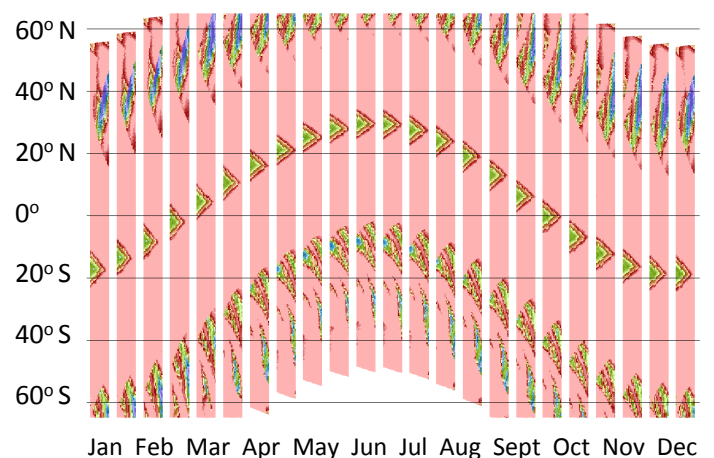

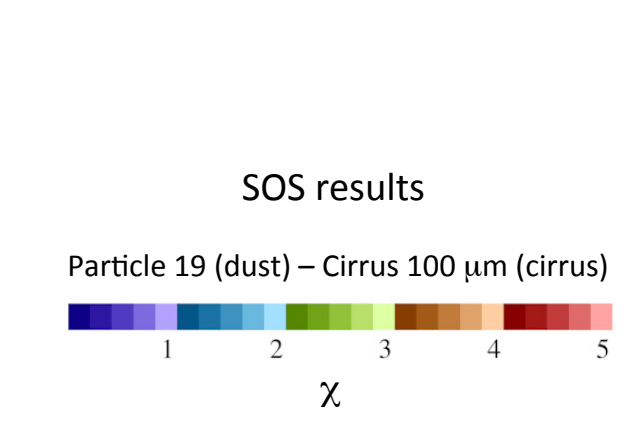

Fig. 18. Same as Fig. 17b but for three additional $\chi^{2}$ metrics used by the MISR V22 Dark Water aerosol retrieval algorithm: (a) $\chi_{\text {spec }}^{2}$, (b) $\chi_{\text {geom }}^{2}$, and (c) $\chi_{\text {maxdev }}^{2}$. These metrics emphasize different aspects of the MISR observing strategy, and different thresholds are employed for these metrics compared to $\chi_{\mathrm{abs}}^{2}$. The V22 thresholds are 3, 3, and 5, for "spec", "geom", and "maxdev", respectively.

model. Now bands of extremely low $\chi_{\mathrm{abs}}^{2}$ are apparent, and the location of two of these bands is consistent with the bands of temporally varying enhanced non-sphericity in the Northern and Southern Hemispheres in Fig. 8. A third band is also present around the subsolar geometry. In this case, the implication is naturally occurring cirrus could be matched with the non-spherical dust model that is included in the existing MISR SMART dataset, producing bands of non-sphericity that are not due to the presence of atmospheric dust.

As described previously, the MISR V22 Dark Water retrieval algorithm employs three additional $\chi^{2}$ metrics that are designed to provide additional particle discrimination (Kahn et al., 1997; Martonchik et al., 1998; Kahn et al., 2001). We briefly examine the performance of these metrics comparing the cirrus atmosphere with an AOD of 0.21 with the reference atmosphere containing Particle 19 with an AOD of 0.10 . The results of the determination of these $\chi^{2}$ metrics are shown in Fig. 18. $\chi_{\text {spec }}^{2}$, which is designed to emphasize spectral differences between the two aerosol models, is shown in Fig. 18a. In this case, the spectral differences between MISR Particle 19 (medium dust) and the cirrus model are small for the red and NIR wavelengths used in the MISR V22 Dark Water aerosol retrieval when the total AOD is less than 0.5, so spectral differences do not provide any additional ability to differentiate between the cirrus and dust aerosol models. $\chi_{\text {geom }}^{2}$, shown in Fig. 18b, is sensitive enough to differences between MISR Particle 19 (medium dust) and the $100 \mu \mathrm{m}$ effective diameter cirrus to distinguish between them for nearly all geometries, with the exception of the subsolar geometry. While this figure shows that the specific cirrus model used in the comparison is distinguishable from the non-spherical dust model, this does not mean another cirrus model, or some combination of cirrus and spherical aerosol, would not yield TOA equivalent reflectances that would produce $\chi_{\text {geom }}^{2}$ values less than 3 when compared with MISR Particle 19. In fact, it is unlikely that naturally occurring cirrus will be identical to the $100 \mu \mathrm{m}$ cirrus model used here. Investigation of these details will be the subject of future work. $\chi_{\text {maxdev }}^{2}$, shown in Fig. 18c, is the maximum deviation parameter. In the figure, bands of reduced sensitivity for $\chi_{\operatorname{maxdev}}^{2}$ appear in the latitude ranges of the enhanced non-spherical bands in Fig. 8, as well as near the subsolar geometry, consistent with reduced sensitivity at these latitudes.

Taken together, the results in Fig. 18 indicate that unscreened cirrus with scattering properties similar to the cirrus model developed by Baum et al. (2005a,b), with effective diameters on the order of $100 \mu \mathrm{m}$, serves as a viable explanation for the appearance of bands of enhanced particle 
non-sphericity in the MISR V22 aerosol climatology. It is unlikely that any spherical aerosol model missing from the MISR SMART could be the underlying cause of these bands because a model designed specifically to mimic the behavior of MISR Particle 19 (medium mode dust) in the backscatter direction does not even pass the $\chi_{\mathrm{abs}}^{2}$ criteria when full multiple-scattering calculations are performed.

\section{Conclusions}

In this study, we examined the sensitivity of the operational MISR V22 Dark Water aerosol retrieval algorithm over the global ocean accounting for changing viewing geometry with latitude and season, as well as the effects of screening specific cameras due to possible contamination from sunglint. We described the non-spherical dust aerosol models that were introduced into the MISR SMART beginning with V16. The introduction of these models improved the overall performance of the MISR aerosol retrieval in dusty regions in terms of both accuracy and coverage. However, artifacts appeared in the global non-spherical AOD fraction climatology as temporally varying bands of enhanced particle nonsphericity in locations where dust aerosols are unlikely to be found. It was demonstrated that these bands did not only occur in low-AOD situations, and a formal analysis approach was described that allowed for investigation of these bands in terms of the temporally changing angular sampling of the MISR instrument over water, including the effects of excluding cameras making observations within $40^{\circ}$ of the specular direction, which could possibly be affected by sunglint. Examination of the particle phase functions showed that aerosol models not currently included in the MISR SMART could be the cause of the bands of enhanced non-sphericity due to their similarity to the non-spherical dust models.

Going through single scattering and full multiplescattering calculations accounting for the actual MISR viewing geometry from the Terra satellite platform, and introducing the $\chi^{2}$ "goodness-of-fit" metrics used in the MISR V22 Dark Water aerosol retrieval algorithm, most of these candidate models were systematically eliminated. However, unscreened cirrus with an effective diameter around $100 \mu \mathrm{m}$ remained a viable candidate for causing artifacts in the MISR non-spherical AOD climatology. This conclusion is consistent with the results of Pierce et al. (2010), who demonstrated that thin cirrus could be retrieved as an aerosol component by the MISR Dark Water retrieval algorithm. More detailed investigation of cirrus models and the effects of including such models in the operational MISR Dark Water aerosol retrieval algorithm will be the subject of future work. Additionally, we plan to investigate whether or not the proposed cirrus particles can be detected in the regions where the MISR V22 Dark Water algorithm retrieves non-spherical dust by examining the MODIS $1.38 \mu \mathrm{m}$ channel, which is sensitive to the presence of high-altitude clouds (Gao et al., 2002), and lidar profiles from ground stations as well as the CALIPSO satellite instrument.

One approach that shows promise for allowing for users to deal with potential cirrus contamination effects in the current MISR V22 Dark Water aerosol product is to consider the $\chi_{\text {abs }}^{2}$ metric, which is reported as the RegLowestResidChisq field. This is the value of $\chi_{\mathrm{abs}}^{2}$ for the aerosol mixture that has the best overall agreement with the MISR observations based on a weighting of all four $\chi^{2}$ parameters. Smaller values of $\chi_{\mathrm{abs}}^{2}$ indicate better agreement with the observations. We have found that constraining $\chi_{\mathrm{abs}}^{2}$ to values less than 0.2 , for total AOD greater than 0.4 , has the effect of nearly eliminating the presence of the artifacts appearing in the MISR global nonspherical AOD fraction climatology, which is consistent with the explanation that the artifacts are caused by dust models in the MISR SMART providing marginally acceptable fits to naturally occurring cirrus particles. A side effect of this approach, however, is a reduction on the overall global coverage of the MISR retrievals. Even so, this reduction in coverage may be acceptable to users who are interested in the highest quality MISR retrievals of aerosol non-sphericity.

In spite of the presence of these seasonally varying bands of enhanced non-spherical AOD fraction and the appearance of large non-spherical AOD fraction in the Southern Ocean, all of which are likely to be due to the presence of unscreened cirrus or other naturally occurring non-spherical aerosols, the MISR non-spherical AOD fraction climatology shows good performance in regions of well-known dust transport. This is especially true over the Atlantic, where MISR aerosol retrievals have been successfully used to study dust transported in the Saharan Air Layer (Kalashnikova and Kahn, 2008). In addition, the MISR aerosol retrievals show sensitivity to dust being transported across the Pacific, but analysis of this dust is complicated by the presence of weather systems that entrain the dust as it is transported, and the particular latitudes and times when this dust transport is most significant are also situations when the MISR Dark Water aerosol retrieval algorithm has reduced ability to distinguish dust from cirrus. However, we have demonstrated that MISR observations are exceedingly sensitive to aerosol non-sphericity, and it is unlikely that this sensitivity has yet been fully exploited in the MISR operational Dark Water aerosol retrievals. Making users aware of some of the strengths and limitations of the MISR aerosol products will allow for them to find new ways to exploit these products for their own needs.

Acknowledgements. This work was carried out at the Jet Propulsion Laboratory, California Institute of Technology, under a contract with the National Aeronautics and Space Administration. The MISR data were obtained from the NASA Langley Research Center Atmospheric Science Data Center.

Edited by: D. Tanré 


\section{References}

Baum, B., Heymsfield, A., Yang, P., and Bedka, S.: Bulk scattering properties for the remote sensing of ice clouds: Part I. Microphysical data and models, J. Appl. Meteorol., 44, 1885-1895, doi:10.1175/JAM2308.1, 2005a.

Baum, B., Yang, P., Heymsfield, A., Platnick, S., King, M., Hu, Y., and Bedka, S.: Bulk scattering properties for the remote sensing of ice clouds: Part II. Narrowband models,, J. Appl. Meteorol., 44, 1896-1911, doi:10.1175/JAM2309.1, 2005 b.

Bevington, P. R. and Robinson, D. K.: Data Reduction and Error Analysis for the Physical Sciences, McGraw Hill (New York), 3rd edn., 2003.

Bodhaine, B. A., Wood, N. B., Dutton, E. G., and Slusser, J. R.: On Rayleigh optical depth calculations, J. Atmos. Ocean. Technol., 16, 1854-1861, 1999.

Bopp, L., Kohfeld, K. E., Quéré, C. L., and Aumont, O.: Dust impact on marine biota and atmospheric $\mathrm{CO}_{2}$ during glacial periods, Paleoceanography, 18, D1046, doi:10.1029/2002PA000810, 2003.

Brindley, H. and Ignatov, A.: Retrieval of mineral aerosol optical depth and size information from Meteosat Second Generation solar reflectance bands, Remote Sens. Environ., 102, 344-363, doi:10.1016/j.rse.2006.02.024, 2006.

Brindley, H., Knippertz, P., Ryder, C., and Ashpole, I.: A critical evaluation of the ability of the Spinning Enhanced Visible and Infrared Imager (SEVIRI) thermal infrared red-green-blue rendering to identify dust events: Theoretical analysis, J. Geophys. Res., 117, D07201, doi:10.1029/2011JD017326, 2012.

Bruegge, C. J., Chrien, N. L., Diner, D. J., Kahn, R. A., and Martonchik, J. V.: MISR radiometric uncertainty analyses and their utilization within geophysical retrievals, Metrologia, 35, 571579, 1998a.

Bruegge, C. J., Duval, V. G., Chrien, N. L., Korechoff, R. P., Gaitley, B. J., and Hochberg, E. B.: MISR prelaunch instrument calibration and characterization results, IEEE Trans. Geosci. Remote Sens., 36, 1186-1198, 1998 b.

Bruegge, C. J., Diner, D. J., Kahn, R. A., Chrien, N., Helmlinger, M. C., Gaitley, B. J., and Abdou, W. A.: The MISR radiometric calibration process, Remote Sens. Environ., 107, 2-11, doi:10.1016/j.rse.2006.07.024, 2007.

Christopher, S. A., Gupta, P., and Haywood, J.: Aerosol optical thicknesses over North Africa: 1. Development of a product for model validation using Ozone Monitoring Instrument, Multiangle Imaging Spectroradiometer, and Aerosol Robotic Network, J. Geophys. Res., 113, D00C04, doi:10.1029/2007JD009446, 2008.

Christopher, S. A., Johnson, B., Jones, T., and Haywood, J.: Vertical and spatial distribution of dust from aircraft and satellite measurements during the GERBILS field campaign, Geophys. Res. Lett., 36, L06806, doi:10.1029/2008GL037033, 2009.

Davies, R.: Fast azimuthally dependent model of the reflection of solar radiation by plane-parallel clouds, Appl. Optics, 19, 250$255,1980$.

DeSouza-Machado, S. G., Strow, L. L., Imbiriba, B., Mcann, K., Hoff, R. M., Hannon, S. E., Martins, J. V., Tanré, D., Deuzé, J. L., Ducos, F., and Torres, O.: Infrared retrievals of dust using AIRS: Comparisons of optical depths and heights derived for a North African dust storm to other collocated EOS ATrain and surface observations, J. Geophys. Res., 115, D15201,
doi:10.1029/2009JD012842, 2010.

Diner, D. J., Beckert, J. C., Reilly, T. H., Bruegge, C. J., Conel, J. E., Kahn, R. A., Martonchik, J. V., Ackerman, T. P., Davies, R., Gerstl, S. A. W., Gordon, H. R., Muller, J.-P., Myneni, R., Sellers, R. J., Pinty, B., and Verstraete, M.: Multi-angle Imaging SpectroRadiometer (MISR) instrument description and experiment overview, IEEE Trans. Geosci. Rem. Sens., 36, 1072-1087, 1998.

Diner, D. J., Beckert, J. C., Bothwell, G., and Rodriguez, J.: Performance of the MISR instrument during its first 20 months in Earth orbit, IEEE Trans. Geosci. Rem. Sens., 40, 1449-1466, 2002.

Diner, D. J., Braswell, B. H., Davies, R., Gobron, N., Hu, J., Jin, Y., Kahn, R. A., Knyazikhin, Y., Loeb, N., Muller, J.-P., Nolin, A. W., Pinty, B., Schaaf, C. B., Seiz, G., and Stroeve, J.: The value of multiangle measurements for retrieving structurally and radiatively consistent properties of clouds, aerosols, and surfaces, Rem. Sens. Environ., 97, 495-518, 2005.

Draine, B. T. and Flatau, J. P.: Discrete-dipole approximation for scattering calculations, J. Opt. Soc. Am., A, 1491-1499, 1994.

Dunion, J. P. and Velden, C. S.: The impact of the Saharan air layer on Atlantic tropical cyclone activity, B. Am. Meteorol. Soc., 85, 353-365, doi:10.1175/BAMS-85-3-353, 2004.

Eguchi, K., Uno, I., Yumimoto, K., Takemura, T., Shimizu, A., Sugimoto, N., and Liu, Z.: Trans-pacific dust transport: integrated analysis of NASA/CALIPSO and a global aerosol transport model, Atmos. Chem. Phys., 9, 3137-3145, doi:10.5194/acp-93137-2009, 2009.

Evans, K. F. and Stephens, G. L.: A new polarized atmospheric radiative transfer model, J. Quant. Spectrosc. Rad. Trans., 46, 413423, 1991.

Formenti, P., Schütz, L., Balkanski, Y., Desboeufs, K., Ebert, M., Kandler, K., Petzold, A., Scheuvens, D., Weinbruch, S., and Zhang, D.: Recent progress in understanding physical and chemical properties of African and Asian mineral dust, Atmos. Chem. Phys., 11, 8231-8256, doi:10.5194/acp-11-8231-2011, 2011.

Frank, T. D., Girolamo, L. D., and Geegan, S.: The spatial and temporal variability of aerosol optical depths in the Mojave Desert of southern California, Rem. Sens. Environ, 107, 54-64, 2007.

Gao, B.-C., Yand, P., Han, W., Li, R.-R., and Wiscombe, W. J.: An algorithm using visible and $1.38-\mu \mathrm{m}$ channels to retrieve cirrus cloud reflectances from aircraft and satellite data, IEEE Trans. Geosci. Rem. Sens., 40, 1659-1668, 2002.

Grant, I. P. and Hunt, G. E.: Solution of radiative transfer problems using the invariant $S_{n}$ method, Mon. Not. R. Astron. Soc., 141, 27-41, 1968.

Hovmöller, E.: The trough-and-ridge diagram, Tellus, 1, 62-66, 1949.

Hsu, N. C., Tsay, S. C., King, M., and Herman, J. R.: Aerosol properties over bright-reflecting source regions, J. Meteorol., 5, 557569, 2004.

Hsu, N. C., Tsay, S. C., King, M., and Herman, J. R.: Deep Blue retrievals of Asian aerosol properties during ACE-Asia, IEEE Trans. Geosci. Remote Sensing, 44, 3180-3195, 2006.

Kahn, R., West, R., McDonald, D., Rheingans, B., and Mishchenko, M.: Sensitivity of multiangle remote sensing observations to aerosol sphericity, J. Geophys. Res., 102, 16861-16870, 1997.

Kahn, R., Banerjee, P., McDonald, D., and Diner, D. J.: Sensitivity of multiangle imaging to aerosol optical depth and to pure-particle size distribution and composition over ocean, J. 
Geophys. Res., 103, 32195-32213, 1998.

Kahn, R., Banerjee, P., and McDonald, D.: The sensitivity of multiangle imaging to natural mixtures of aerosols over ocean, J. Geophys. Res., 106, 18219-18238, 2001.

Kahn, R. A., Gaitley, B., Martonchik, J., Diner, D., Crean, K., and Holben, B.: Multiangle Imaging SpectroRadiometer (MISR) global aerosol optical depth validation based on 2 years of coincident Aerosol Robotic Network (AERONET) observations, J. Geophys. Res., 110, D10S04, doi:10.1029/2004JD004706, 2005a.

Kahn, R. A., Li, W.-H., Martonchik, J. V., Bruegge, C. J., Diner, D. J., Gaitley, B. J., Abdou, W., Dubovik, O., Holben, B., Smirnov, A., Jin, Z., and Clark, D.: MISR calibration and implications for low-light-level aerosol retrieval over dark water, J. Atmos. Sci., 62, 1032-1052, $2005 b$.

Kahn, R. A., Garay, M. J., Nelson, D. L., Yau, K. K., Bull, M. A., Gaitley, B. J., Martonchik, J. V., and Levy, R. C.: Satellite-derived aerosol optical depth over dark water from MISR and MODIS: Comparisons with AERONET and implications for climatological studies, J. Geophys. Res., 112, D18205, doi:10.1029/2006JD008175, 2007a.

Kahn, R. A., Li, W. H., Moroney, C., Diner, D. J., Martonchik, J. V., and Fishbein, E.: Aerosol source plume physical characteristics from space-based multiangle imaging, J. Geophys. Res., 112, D11205, doi:10.1029/2006JD007647, 2007b.

Kahn, R. A., Nelson, D. L., Garay, M. J., Levy, R. C., Bull, M. A., Diner, D. J., Martonchik, J. V., Paradise, S. R., Hansen, E. G., and Remer, L. A.: MISR aerosol product attributes and statistical comparisons with MODIS, IEEE Trans. Geosci. Remote Sensing, 47, 4095-4114, 2009a.

Kahn, R., Petzold, A., Wendisch, M., Bierwirth, E., Dinter, T., Esselborn, M., Fiebig, M., Heese, B., Knippertz, P., Muller, D., Schladitz, A., and von Hoyningen-Huene, W.: Desert dust aerosol air mass mapping in the western Sahara, using particle properties derived from space-based multi-angle imaging, Tellus, 61, 239-251, 2009b.

Kahn, R. A., Gaitley, B. J., Garay, M. J., Diner, D. J., Eck, T. F., Smirnov, A., and Holben, B. N.: Multiangle Imaging SpectroRadiometer global aerosol product assessment by comparison with the Aerosol Robotic Network, J. Geophys. Res., 115, D23209, doi:10.1029/2010JD014601, 2010.

Kalashnikova, O. V. and Kahn, R.: Ability of multiangle remote sensing observations to identify and distinguish mineral dust types: 2. Sensitivity over dark water, J. Geophys. Res., 111, D11207, doi:10.1029/2005JD006756, 2006.

Kalashnikova, O. V. and Kahn, R. A.: Mineral dust plume Evolution over the Atlantic from combined MISR/MODIS aerosol retrievals, J. Geophys. Res., 113, D24204, doi:10.1029/2008JD010083, 2008.

Kalashnikova, O. V. and Sokolik, I. N.: Modeling the radiative properties of nonspherical soil-derived mineral aerosols, J. Quant. Spectrosc. Rad. Trans., 87, 137-166, 2004.

Kalashnikova, O. V., Kahn, R., Sokolik, I. N., and Li, W.-H.: The ability of multiangle remote sensing observations to identify and distinguish mineral dust types: Optical models and retrievals of optically thick plumes, J. Geophys. Res., 110, D18S14, doi:10.1029/2004JD004550, 2005.
Kalashnikova, O. V., Garay, M. J., Davis, A. B., Diner, D. J., and Martonchik, J. V.: Sensitivity of multi-angle photo-polarimetry to vertical layering and mixing of absorbing aerosols: Quanitfying measurement uncertainties, J. Quant. Spectrosc. Rad. Trans., 112, 2149-2163, doi:10.1016/j.jqsrt.2011.05.010, 2011.

Kaufman, Y. J., Koren, I., Remer, L. A., Tanré, D., Ginoux, P., and Fan, S.: Dust transport and deposition observed from the Terra-Moderate Resolution Imaging Spectroradiometer (MODIS) spacecraft over the Atlantic Ocean, J. Geophys. Res. 110, D10S12, doi:10.1029/2003JD004436, 2005.

Kinne, S., Schulz, M., Textor, C., Guibert, S., Balkanski, Y., Bauer, S. E., Berntsen, T., Berglen, T. F., Boucher, O., Chin, M., Collins, W., Dentener, F., Diehl, T., Easter, R., Feichter, J., Fillmore, D., Ghan, S., Ginoux, P., Gong, S., Grini, A., Hendricks, J., Herzog, M., Horowitz, L., Isaksen, I., Iversen, T., Kirkevåg, A., Kloster, S., Koch, D., Kristjansson, J. E., Krol, M., Lauer, A., Lamarque, J. F., Lesins, G., Liu, X., Lohmann, U., Montanaro, V., Myhre, G., Penner, J., Pitari, G., Reddy, S., Seland, O., Stier, P., Takemura, T., and Tie, X.: An AeroCom initial assessment - optical properties in aerosol component modules of global models, Atmos. Chem. Phys., 6, 1815-1834, doi:10.5194/acp-6-1815-2006, 2006.

Klüser, L., Kleiber, P., Holzer-Popp, T., and Grassian, V. H.: Desert dust observation from space - Application of measured mineral component infrared extinction spectra, Atmos. Environ., 54, 419-427, 2012.

Koven, C. D. and Fung, I.: Identifying global dust source areas using high-resolution land surface form, J. Geophys. Res., 113, D22204, doi:10.1029/2008JD010195, 2008.

Lallart, P., Kahn, R., and Tanré, D.: POLDER2/ADEOSII, MISR, and MODIS/Terra reflectance comparisons, J. Geophys. Res., 113, D14S02, doi:10.1029/2007JD009656, 2008.

Liou, K. N.: An Introduction to Atmospheric Radiation, Second Edition, Academic Press, 2002.

Lyapustin, A., Wang, Y., Kahn, R., Xiong, J., Ignatov, A., Wolfe, R., $\mathrm{Wu}, \mathrm{A}$., Holben, B., and Bruegge, C.: Analysis of MODIS-MISR calibration differences using surface albedo around AERONET sites and cloud reflectance, Remote Sens. Environ., 107, 12-21, 2007.

Mahowald, N. M., Engelstaedter, S., Luo, C., Sealy, A., Artaxo, P., Benitez-Nelson, C., Bonnet, S., Chen, Y., Chuang, P. Y., Cohen, D. D., Dulac, F., Herut, B., Johansen, A. M., Kubilay, N., Losno, R., Maenhaut, W., Paytan, A., Prospero, J. M., Shank, L. M., and Seifert, R. L.: Atmospheric iron deposition: Global distribution, variability and human perturbations, Ann. Rev. Mar. Sci., 1, 245278, doi:10.1146/annurev.marine.010908.163727, 2009.

Martin, J. H., Fitzwater, S. E., and Gordon, R. M.: We still say iron deficiency limits phytoplankton growth in the Subarctic Pacific, J. Geophys. Res.,, 96, 20699-20700, 1991.

Martonchik, J. V., Diner, D. J., Kahn, R. A., Ackerman, T. P., Verstraete, M. M., Pinty, B., and Gordon, H. R.: Techniques for the retrieval of aerosol properties over land and ocean using multiangle imaging, IEEE Trans. Geosci. Remote Sens., 36, 1212-1227, 1998.

Martonchik, J., Diner, D., Crean, K., and Bull, M.: Regional Aerosol Retrieval Results From MISR, IEEE Trans. Geosci. Remote Sens., 40, 1520-1531, 2002. 
Martonchik, J. V., Diner, D. J., Kahn, R., Gaitley, B., and Holben, B. N.: Comparison of MISR and AERONET aerosol optical depths over desert sites, Geophys. Res. Lett., 31, L16102, doi:10.1029/2004GL019807, 2004.

Martonchik, J. V., Kahn, R., and Diner, D. J.: Retrieval of Aerosol Properties over Land Using MISR Observations, in: Satellite Aerosol Remote Sensing Over Land, edited by: Kokhanovsky, A. A. and de Leeuw, G., 31, Springer, Berlin, 2009.

Mishchenko, M. I., Travis, L. D., and Mackowski, D. W.: T-matrix computations of light scattering by nonspherical particles: A Review, J. Quan. Spec. Rad. Trans., 55, 535-575, 1996.

Ottaviani, M., Spurr, R., Stamnes, K., Li, W., Su, W., and Wiscombe, W.: Improving the description of sunglint for accurate prediction of remotely sensed radiances, J. Quan. Spec. Rad. Trans., 109, 2364-2375, doi:10.1016/j.jqsrt.2008.05.012, 2008.

Peyridieu, S., Chédin, A., Capelle, V., Tsamalis, C., Pierangelo, C., Armante, R., Crevoisier, C., Crépeau, L., Siméon, M., Ducos, F., and Scott, N. A.: Characterisation of dust aerosols in the infrared from IASI and comparison with PARASOL, MODIS, MISR, CALIOP, and AERONET observations, Atmos. Chem. Phys., 13, 6065-6082, doi:10.5194/acp-13-6065-2013, 2013.

Pierce, J. R., Kahn, R. A., Davis, M. R., and Comstock, J. M.: Detecting thin cirrus in Multiangle Imaging Spectroradiometer aerosol retrievals, J. Geophys. Res., 115, D08201, doi:10.1029/2009JD013019, 2010.

Russell, P. B., Livingston, J. M., Dutton, E. G., Pueschel, R. F., Reagan, J. A., and Defoor, T. E.: Pinatubo and pre-Pinatubo opticaldepth spectra: Mauna Loa measurements, comparisons, inferred particle size distributions, radiative effects, and relationship to lidar data, J. Geophys. Res., 98, 22969-22985, 1993.

Sayer, A. M., Hsu, N. C., Bettenhausen, C., Jeong, M.-J., Holben, B. N., and Zhang, J.: Global and regional evaluation of overland spectral aerosol optical depth retrievals from SeaWiFS, Atmos. Meas. Tech., 5, 1761-1778, doi:10.5194/amt-5-1761-2012, 2012.

Schladits, A., Muller, T., Massling, A., Kaaden, N., Kandler, K., and Wiedensohler, A.: In situ measurements of optical properties at Tinfou (Morocco) during the Saharan mineral dust experiment, SAMUM, Tellus, 61, 64-78, doi:10.1111/j.16000889.2008.00397.x, 2009.

Shao, Y. and Dong, C. H.: A review on East Asian dust storm climate, modelling and monitoring, Global and Planetary Change, 52, 1-22, 2006.
Shi, Y., Zhang, J., Reid, J. S., Hyer, E. J., Eck, T. F., Holben, B. N., and Kahn, R. A.: A critical examination of spatial biases between MODIS and MISR aerosol products - application for potential AERONET deployment, Atmos. Meas. Tech., 4, 2823 2836, doi:10.5194/amt-4-2823-2011, 2011.

Shinn, E. A., Smith, G. W., Prospero, J. M., Betzer, P., Hayes, M. L., Garrison, V., and Barber, R. T.: African dust and the demise of Caribbean coral reefs, Geophys. Res. Lett., 27, 302-3032, 2000.

Sokolik, I. N., Winker, D. M., Bergametti, G., Gillette, D. A., Carmichael, G., Kaufman, Y. J., Gomes, L., Schuetz, L., and Penner, J. E.: Introduction to special section: Outstanding problems in quantifying the radiative impacts of mineral dust, J. Geophys. Res., 106, 18015-18028, 2001.

Sun, J., Zhang, M., and Liu, T.: Spatial and temporal characteristics of dust storms in China and its surrounding regions, 19601999: Relations to source area and climate, J. Geophys. Res., 106, 10325-10333, 2001.

Uno, I., Eguchi, K., Yumimoto, K., Takemura, T., Shimizu, A., Uematsu, M., Liu, Z., Wang, Z., Hara, Y., and Sugimoto, N.: Asian dust transported one full circuit around the globe, Nature Geosci., 2, 557-560, 2009.

Wang, Z., Akimoto, H., and Uno, I.: Neutralization of soil aerosol and its impact on the distribution of acid rain over east Asia: Observations and model results, J. Geophys. Res., 107, 4389, doi:10.1029/2001JD001040, 2002.

Yu, H., Remer, L. A., Chin, M., Bian, H., Tan, Q., Yuan, T., and Zhang, Y.: Aerosols from overseas rival domestic emissions over North America, Science, 337, 566-569, doi:10.1126/science.1217576, 2012.

Zhai, P. W., Hu, Y., Trepte, C. R., and Lucker, P. L.: A vector radiative transfer model for coupled atmosphere and ocean systems based on successive order of scatter method, Opt. Expr., 17, 2057-2079, 2009.

Zhai, P.-W., Hu, Y., Chowdhary, J., Trepte, C. R., Lucker, P. L., and Josset, D.: A vector radiative transfer model for coupled atmosphere and ocean systems with a rough interface, J. Quan. Spec. Rad. Trans., 111, 1025-1040, doi:10.1016/j.jqsrt.2009.12.005, 2010.

Zhang, J. and Reid, J. S.: A decadal regional and global trend analysis of the aerosol optical depth using a data-assimilation grade over-water MODIS and Level 2 MISR aerosol products, Atmos. Chem. Phys., 10, 10949-10963, doi:10.5194/acp-1010949-2010, 2010. 